\title{
Selective binding of small molecules to Vibrio cholerae DsbA offers a starting point for the design of novel antibacterials
}

Geqing Wang ${ }^{\mathrm{a},{ }^{*}}$, Biswaranjan Mohanty,h,, , Martin L. Williams, ${ }^{\mathrm{b}, *}$, Bradley C. Doak ${ }^{\mathrm{b},{ }^{*}}$, Rabeb Dhouib $^{\mathrm{c}}$, Makrina Totsika ${ }^{\mathrm{c}}$, Róisín M. McMahon ${ }^{\mathrm{d}}$, Gaurav Sharma ${ }^{\mathrm{b}}$, Dan Zheng ${ }^{\mathrm{b}}$, Matthew R. Bentley $^{\mathrm{b}}$, Yanni Ka-Yan Chin ${ }^{\mathrm{e}, \mathrm{f}}$, James Horne ${ }^{\mathrm{g}}$, David K. Chalmers ${ }^{\mathrm{b}}$, Begoña Heras ${ }^{\mathrm{a}, \#}$, Martin J. Scanlon ${ }^{\mathrm{b}, \mathrm{h}, \#}$

aDepartment of Biochemistry and Genetics, La Trobe Institute for Molecular Science, La Trobe University, Melbourne, Victoria, Australia.

${ }^{b}$ Medicinal Chemistry, Monash Institute of Pharmaceutical Sciences, Monash University, Victoria, Australia.

${ }^{c}$ Queensland University of Technology, Centre for Immunology and Infection Control, School of Biomedical Sciences, Brisbane, Queensland, Australia

${ }^{\mathrm{d}}$ Griffith Institute for Drug Discovery, Griffith University, Nathan, QLD, 4111, Australia Institute for Molecular Bioscience, The University of Queensland, St Lucia, QLD 4072, Australia

${ }^{f}$ Centre for Advanced Imaging, The University of Queensland, St Lucia, QLD 4072, Australia gCentral Science Laboratory, The University of Tasmania, Sandy Bay, TAS, 7005, Australia ${ }^{\mathrm{h}}$ ARC Training Centre for Fragment Based Design, Monash Institute of Pharmaceutical Sciences, Monash University, 381 Royal Parade, Parkville, VIC 3052, Australia

\footnotetext{
*Authors contributed equally to this work.

\#Address correspondence to Begoña Heras (b.heras@latrobe.edu.au) ORCID 0000-0003-34697988 and Martin J. Scanlon (martin.scanlon@monash.edu) ORCID 0000-0002-9230-7506
} 


\section{Abstract}

DsbA enzymes catalyze oxidative folding of proteins that are secreted into the periplasm of Gram-negative bacteria, and they are indispensable for the virulence of human pathogens such as Vibrio cholerae and Escherichia coli. Therefore, targeting DsbA represents an attractive approach to control bacterial virulence. X-ray crystal structures reveal that DsbA enzymes share a similar fold, however, the hydrophobic groove adjacent to the active site, which is implicated in substrate binding, is shorter and flatter in the structure of $V$. cholerae DsbA (VcDsbA) compared to E. coli DsbA (EcDsbA). The flat and largely featureless nature of this hydrophobic groove is challenging for the development of small molecule inhibitors. Using fragment-based screening approaches, we have identified a novel small molecule, based on the benzimidazole scaffold, that binds to the hydrophobic groove of oxidized VcDsbA with a $K_{\mathrm{D}}$ of $446 \pm 10 \mu \mathrm{M}$. The same benzimidazole compound has $\sim 8$-fold selectivity for VcDsbA over EcDsbA and binds to oxidized EcDsbA, with $K_{\mathrm{D}}>3.5 \mathrm{mM}$. We generated a model of the benzimidazole complex with $\mathrm{VcDsbA}$ using NMR data but were unable to determine the structure of the benzimidazole bound EcDsbA using either NMR or X-ray crystallography. Therefore, a structural basis for the observed selectivity is unclear. To better understand ligand binding to these two enzymes we crystallized each of them in complex with a known ligand, the bile salt sodium taurocholate. The crystal structures show that taurocholate adopts different binding poses in complex with $\mathrm{VcDsbA}$ and $\mathrm{EcDsbA}$, and reveals the protein-ligand interactions that stabilize the different modes of binding. This work highlights the capacity of fragment-based drug discovery to identify inhibitors of challenging protein targets. In addition, it provides a starting point for development of more potent and specific VcDsbA inhibitors that act through a novel anti-virulence mechanism.

\section{Introduction}

Vibrio cholerae is a Gram-negative bacterium that causes cholera in humans. Cholera is an acute diarrhoeal disease with an estimated burden of 1.3 to 4.0 million cases and 21,000 to 143,000 deaths per year worldwide. ${ }^{[1]}$ Infection with $V$. cholerae is most commonly acquired through the ingestion of contaminated food or water. ${ }^{[2]}$ The virulence of $V$. cholerae is attributable to its deployment of multiple virulence factors, including cholera toxin (CT) and the toxin-coregulated pilus (TCP). ${ }^{[2 b]} \mathrm{CT}$ acts on enterocytes, causing efflux of ions and water from the cells, leading to watery diarrhoea. TCPs promote host colonization by adhering to host microvilli and are pivotal to the pathogenesis of $V$. cholerae. ${ }^{[3]}$ The conformational 
maturation and secretion of virulence factors, including CT and TCP, depend on oxidative folding in the periplasm, which is catalyzed by the thiol-disulfide oxidoreductase enzyme, $V$. cholerae DsbA (VcDsbA), encoded by the tcpg gene. ${ }^{[4]}$ Disruption of VcDsbA leads to defects in the functional folding and secretion of $V$. cholerae virulence factors. This, in turn has multiple phenotypic effects in $V$. cholerae including compromised colonization and autoagglutination. ${ }^{[4-5]}$ Therefore, inhibitors of $\mathrm{VcDsbA}$ may attenuate the virulence of $V$. cholerae.

DsbA enzymes from other Gram-negative bacteria have been identified as key mediators of bacterial virulence and may therefore represent promising antibacterial drug targets. In many cases it has been shown that bacteria lacking a functional DsbA enzyme have reduced virulence, enhanced sensitivity to antibiotics and decreased capacity to initiate infection. ${ }^{[6]}$ For example, deletion of $d s b A$ in uropathogenic $E$. coli (UPEC) strongly attenuated its ability to colonize the bladder ${ }^{[7]}$ and $d s b A$ mutants rendered Salmonella enterica serovar Typhimurium avirulent. ${ }^{[8]}$ DsbA enzymes catalyze disulfide bond formation in the periplasm of Gram-negative bacteria. The loss of virulence in $d s b A$ mutants can often be linked to the misfolding of a DsbA substrate. ${ }^{[9]}$

DsbA enzymes retain a conserved tertiary structure, comprising a classical thioredoxin (TRX) domain with an inserted $\alpha$-helical domain (Fig. 1A-B). The TRX domain contains a pair of redox-active cysteine residues in a CXXC motif located adjacent to a conserved cis-proline residue (Fig. 1A). ${ }^{[10]}$ These features contribute to a hydrophobic patch of residues located in a surface groove of DsbA that is required for substrate recognition. ${ }^{[11]}$ In the oxidative folding reaction, cysteine residues at the active site of DsbA cycle between their oxidized (disulfide) and reduced (dithiol) state. Oxidized DsbA reacts with reduced protein substrates to form a mixed disulfide intermediate. ${ }^{[12]}$ This covalent intermediate is resolved to release the oxidized substrate and reduced DsbA. To prime for the next redox cycle, the active site cysteine pair of reduced DsbA is re-oxidized by the inner membrane protein DsbB in concert with its cofactor ubiquinone, restoring DsbA to its active oxidized state. ${ }^{[13]}$

The hydrophobic groove of DsbA enzymes mediates interaction with both DsbB and substrates. The relatively flat and featureless surface of the groove represents a significant obstacle for inhibitor design (Fig. 1C-D). ${ }^{[14]}$ Nonetheless, small molecule inhibitors targeting DsbA enzymes have been identified. ${ }^{[15]}$ Covalent peptide inhibitors of E. coli DsbA (EcDsbA) have been developed based on the sequence of the loop in EcDsbB that interacts directly with 
EcDsbA. ${ }^{[16]}$ Additionally, small molecule inhibitors of EcDsbA have been developed using fragment-based screening methods. ${ }^{[15 b, 15 f, 16]}$

Although VcDsbA shares the canonical DsbA fold, there are some differences between the active sites of VcDsbA and EcDsbA (Fig. 1). In particular VcDsbA possesses a more truncated and flatter groove compared to EcDsbA. ${ }^{[17]}$ In this work, we sought to identify small molecule inhibitors of VcDsbA using a fragment-based screening strategy and to characterize their binding to VcDsbA. In addition, to examine difference in ligand recognition between these two enzymes, structures of their complexes with a known ligand, the bile salt sodium taurocholate (TC), ${ }^{[18]}$ were characterized by X-ray crystallography. Together these structural and binding data highlight important differences between EcDsbA and VcDsbA in the context of inhibitor design. In addition, the compounds described here provide starting points for the future development of more potent $\mathrm{VcDsbA}$ inhibitors.

\section{Results}

\section{Fragment-based discovery of VcDsbA inhibitors}

To identify small molecules that bound to oxidized VcDsbA, a library of 500 fragments from Maybridge (ThermoFisher Scientific) was screened by 1D ${ }^{1} \mathrm{H}$ Saturation Transfer Difference (STD) NMR spectroscopy. Fifteen fragments were identified as hits (Fig. S1) based on the intensity of signals observed in their STD spectra ${ }^{[19]}$ and their binding was confirmed by recording ${ }^{1} \mathrm{H}-{ }^{15} \mathrm{~N}$ HSQC spectra of VcDsbA in the absence and presence of each fragment. The most promising hit was the trifluoromethyl-substituted benzimidazole 1 (Fig. 2), which elicited chemical shift perturbations (CSP) of peaks corresponding to residues located in the hydrophobic groove of $\mathrm{VcDsbA}$ (Fig. S2A). The binding affinity was estimated by recording ${ }^{1} \mathrm{H}-{ }^{15} \mathrm{~N}$ HSQC spectra in the presence of increasing concentrations of benzimidazole 1 , which yielded an equilibrium dissociation constant $\left(K_{\mathrm{D}}\right)$ of $610 \pm 150 \mu \mathrm{M}$. To generate preliminary structure-binding relationships, seven benzimidazole analogues were tested for their ability to bind to VcDsbA as listed in Supplementary Figure S2. The analogues were designed to evaluate which portions of benzimidazole 1 were essential for binding, and to identify positions that could be developed to improve affinity. The binding of these analogues was evaluated by monitoring the extent of CSP observed in ${ }^{1} \mathrm{H}-{ }^{15} \mathrm{~N}$ HSQC upon addition of the compounds (1 $\mathrm{mM})$. Two of the seven analogues elicited larger CSP in ${ }^{1} \mathrm{H}-{ }^{15} \mathrm{~N}$ HSQC of VcDsbA than the parent benzimidazole, suggesting that they bound with higher affinity (Fig. S2). Benzimidazole 
2 (Fig 2B) gave the largest CSP in the HSQC spectra and was selected for additional characterization.

To map the binding site, ${ }^{15} \mathrm{~N}$-labeled oxidized $\mathrm{VcDsbA}(100 \mu \mathrm{M})$ was titrated with benzimidazole $2(0.05 \mathrm{mM}$ to $3 \mathrm{mM})$ at $298 \mathrm{~K}$. The CSP observed in the ${ }^{1} \mathrm{H}-{ }^{15} \mathrm{~N}$ HSQC spectra of $\mathrm{VcDsbA}$ showed linear perturbations with increasing ligand concentration, consistent with 1:1 binding stoichiometry and indicating that the complex was in fast exchange on the NMR chemical shift time scale. Residues with a CSP $>0.05 \mathrm{ppm}$ at the highest ligand concentration tested were mapped onto the VcDsbA structure (Fig. 2C, D). The residues showing the largest CSP were localized within the hydrophobic groove, adjacent to the active site (Fig. 2D). The CSP titration data were fitted to a one-site binding model, which yielded a $K_{\mathrm{D}}$ of $446 \pm 10 \mu \mathrm{M}$ for the VcDsbA-benzimidazole 2 interaction (Fig. 2E). The $K_{\mathrm{D}}$ for the VcDsbAbenzimidazole 2 interaction was also estimated using ligand-detected $1 \mathrm{D}{ }^{19} \mathrm{~F}-\mathrm{NMR}$ (Fig. 2F). The ${ }^{19} \mathrm{~F}$ chemical shift of the $\mathrm{CF}_{3}$ group in benzimidazole 2 has a chemical shift of $-60.38 \mathrm{ppm}$ in the absence of protein and was perturbed upon in a concentration-dependent fashion upon addition of VcDsbA. The change in the chemical shift of the ${ }^{19} \mathrm{~F}$ signal at two different protein:ligand ratios was used to estimate the $K_{\mathrm{D}}$ of the interaction. ${ }^{[20]}$ This analysis produced a value of $K_{\mathrm{D}}=480 \mu \mathrm{M}$ (Fig. $2 \mathrm{~F}$ ), which is in good agreement with the value calculated from the ${ }^{1} \mathrm{H}-{ }^{15} \mathrm{~N}$ HSQC titrations.

Since benzimidazole 2 binds into the hydrophobic groove of VcDsbA, we also evaluated its ability to bind to the corresponding hydrophobic groove in EcDsbA, which is larger than that of $\mathrm{VcDsbA}$. HSQC titration showed that benzimidazole 2 binds with low affinity to the hydrophobic groove of oxidized EcDsbA. The titration did not reach saturation at the highest concentration of benzimidazole 2 that was tested, and analysis of the data revealed that it bound with a $K_{\mathrm{D}}$ of $>3.5 \mathrm{mM}$ (Fig. S3), at least 8-fold weaker than its affinity to VcDsbA. The pattern of CSP in the HSQC spectra of EcDsbA upon titration with benzimidazole 2 was slightly different to that observed for VcDsbA. In addition to the CSP observed for residues in the hydrophobic groove, a cluster of residues on the opposite face of EcDsbA were also perturbed, suggesting benzimidazole 2 may bind both to the hydrophobic groove and in the cleft between the two domains of EcDsbA. Small molecules have previously been identified that bind in a

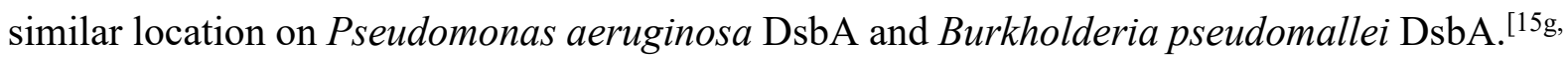
19] 


\section{Characterization of benzimidazole 2 binding mode with VcDsbA}

To understand the selectivity of benzimidazole 2 binding, we next sought to generate structures in complex with VcDsbA and EcDsbA. Attempts to soak and co-crystallize benzimidazole 2 with $\mathrm{VcDsbA}$ and EcDsbA were unsuccessful, and no electron density of the compound was observed in the resulting crystal structures. Therefore we determined an NMR model of the complex with VcDsbA using a previously described protocol. ${ }^{[21]}$ The solubility of benzimidazole 2 in aqueous NMR buffer was established by recording a $2 \mathrm{D}\left[{ }^{1} \mathrm{H},{ }^{1} \mathrm{H}\right]-\mathrm{NOESY}$ spectrum (NOE mixing time of $800 \mathrm{~ms}$ ) which revealed that NOE cross peaks were the opposite phase to the diagonal. This observation suggests that benzimidazole 2 was soluble and free from aggregation at the concentration of $2.5 \mathrm{mM}$ used in this experiment (Fig. S4). A 3D $\omega_{1-}$ ${ }^{13} \mathrm{C},{ }^{15} \mathrm{~N}$-filtered, $\omega_{3}{ }^{13} \mathrm{C}_{\mathrm{ali}}$ (methyl) edited $\left[{ }^{1} \mathrm{H},{ }^{1} \mathrm{H}\right]$-NOESY was acquired using a small spectral width selecting only the methyl region in the indirect carbon dimension, ${ }^{[22]}$ on a sample containing uniformly ${ }^{13} \mathrm{C},{ }^{15} \mathrm{~N}$-labelled VcDsbA $(0.25 \mathrm{mM})$ and unlabeled benzimidazole 2 (2.5 $\mathrm{mM})$ with $2 \%(v / v) \mathrm{d}_{6}$-DMSO. We observed a total of 21 intermolecular NOEs between oxidized VcDsbA and benzimidazole 2 (Table S2). NOEs were observed to the methyl resonances of I39, V151, V159 and L167 of VcDsbA (Fig. 3B). No intermolecular NOEs were detected to A164, which is the only methyl-containing residue in the dynamic loop L3 of the VcDsbA hydrophobic groove. Using the intermolecular NOEs and ${ }^{1} \mathrm{H}^{-15} \mathrm{~N}$ HSQC CSP as ambiguous interaction restraints (AIR), we generated models of the VcDsbA-benzimidazole 2 complex using HADDOCK, ${ }^{[23]}$ using a protocol described previously. ${ }^{[21]}$ A final set of 10 conformers was selected to represent the structure of the complex, which had the lowest HADDOCK scores and restraint violation energies. In all models, benzimidazole 2 was found in a similar orientation. The largest cluster comprising 5 out of 10 conformers is shown in Fig. 3C-D. In this NMR model, the benzimidazole 2 core and chlorine group make hydrophobic and $\pi-\pi$ interactions to $\mathrm{F} 36, \mathrm{I} 39, \mathrm{~L} 167$, and Y170 while the $\mathrm{CF}_{3}$ group makes hydrophobic contacts with V151 and V159.

\section{Characterizing the binding site and binding interactions of TC to oxidized VcDsbA and EcDsbA by NMR spectroscopy}

Xue et al. have reported that TC binds to VcDsbA and EcDsbA with $K_{\mathrm{D}}$ values of $40 \pm 2.5 \mu \mathrm{M}$ and $146 \pm 7 \mu \mathrm{M}$, respectively, as measured by ITC. ${ }^{[18]}$ The same study reported that TC was able to affect the redox state of $\mathrm{VcDsbA}$ and inhibit $\mathrm{VcDsbA}$ substrate oxidation in vivo, ${ }^{[18]}$ suggesting TC may bind to the hydrophobic groove. To characterize the binding site and 
binding modes of TC, we characterized the interaction of TC with EcDsbA and VcDsbA by NMR and ITC and, in parallel solved the crystal structures of TC bound to EcDsbA and VcDsbA.

\section{NMR studies of TC binding}

Uniformly ${ }^{15} \mathrm{~N}$-labeled oxidized VcDsbA $(100 \mu \mathrm{M})$ and EcDsbA $(95 \mu \mathrm{M})$ were titrated with increasing concentrations of TC at $298 \mathrm{~K}$ (Figs. 4 and 5). The solubility and quality of TC have been confirmed by $1 \mathrm{D}{ }^{1} \mathrm{H}$ NMR and 2D ${ }^{1} \mathrm{H}-{ }^{1} \mathrm{H}$ NOESY (Figs. S5 and S6). The ${ }^{1} \mathrm{H}-{ }^{15} \mathrm{~N}$ HSQC spectra revealed that TC perturbed residues in the hydrophobic groove and the active site of VcDsbA (Fig. 4B, C). TC also shifted residues in the hydrophobic groove of EcDsbA (Fig. 5A, B), producing similar patterns of CSP with each enzyme (Fig. 4B, C vs. 5A, B). CSP were mapped onto each DsbA crystal structure as illustrated in Figs. 4E and 5D. Binding affinities for TC were measured by ${ }^{1} \mathrm{H}^{15} \mathrm{~N}$ HSQC-NMR titrations, from which $K_{\mathrm{D}}$ values were determined to be $148 \pm 6.5 \mu \mathrm{M}$ for VcDsbA and $224 \pm 5 \mu \mathrm{M}$ for EcDsbA (Fig. 4D and Fig. 5C). ITC measurements yielded $K_{\mathrm{D}}$ values of $112 \pm 11 \mu \mathrm{M}$ and $428 \pm 44 \mu \mathrm{M}$ for $\mathrm{VcDsbA}$ and EcDsbA, respectively (Fig. S7). The discrepancies between the NMR and ITC-derived values is not unexpected for low affinity interactions such as these. The larger differences between these values and those reported by Xue et al. may reflect the different solution conditions used in the respective studies.

These data confirm that TC binds to the hydrophobic groove of both DsbA enzymes, and with slightly higher affinity in the case of VcDsbA. Although it has been suggested that high concentrations of bile salts may cause protein unfolding, ${ }^{[24]}$ there is no evidence of this in the HSQC spectra for either protein even at the highest TC concentration tested $(\sim 1.7 \mathrm{mM})$. In both cases resonances show linear CSP upon titration with TC, and peaks with CSP $>0.05 \mathrm{ppm}$ correspond to residues around the hydrophobic groove, suggesting two-state exchange consistent with 1:1 binding. The NMR data also revealed that, under the conditions used for the titrations, the TC did not induce protein unfolding and had no effect on the oxidation state of either DsbA, as there was no indication of resonances characteristic of the reduced form of either protein.

\section{Crystal structures of TC in complex with EcDsbA and VcDsbA}


To identify the structural details of the interaction between TC and DsbA, we solved the crystal structures of TC in complex with oxidized EcDsbA and VcDsbA using molecular replacement at resolutions of $1.79 \AA$ and $1.74 \AA$, respectively (Table S1). Crystals were obtained by cocrystallization and each asymmetric unit contained a single DsbA molecule with TC bound (Fig. 6 and Fig. 7) as confirmed by omit maps (Fig. 6D, 7D, S9).

In the complex with EcDsbA, TC occupies the full length of the hydrophobic groove and buries $435 \AA^{2}$ of solvent accessible surface area (SASA). It interacts with H32 in the active site and cis-P151, both of which are highly conserved in DsbA enzymes and critical for disulfide catalysis (Fig. 6B). The interaction with the steroid core at the right-hand side of the groove (proximal to the active site) is driven by hydrophobic interactions with F36, L40, M171, F174, P163 and L161. On the left-hand side (distal to the active site), the negatively charged sulfonate group of TC forms a network of polar interactions with the side chain amide of Q160 and a series of water molecules, and its amide NH forms a hydrogen bond with the backbone amide of $c i s-\mathrm{P} 151$. The binding of TC does not cause major conformational change of EcDsbA, which is also generally the case in previously reported complexes with other small molecules. ${ }^{[15 e, 16 \text {, }}$ ${ }^{25]}$ Additional polar contacts are made to a symmetry related EcDsbA molecule through the amide carbonyl and hydroxyl groups of TC which point away from the hydrophobic groove (Fig. S8), however no hydrophobic interactions are made to the symmetry related EcDsbA molecule and the solution HSQC NMR data are consistent with the binding mode determined by X-ray crystallography with significant CSP located in the hydrophobic groove.

TC also binds to the hydrophobic groove of VcDsbA but adopts a different binding pose compared to the complex with EcDsbA (Fig. 7A). The binding contributes to a buried SASA of $401 \AA^{2}$, which is similar to that of the EcDsbA-TC complex. Only partial electron density of TC was observed at $1 \sigma$, nevertheless full electron density can be clearly revealed if the $2 \mathrm{~F}_{0^{-}}$ $F_{c}$ map is contoured at $0.7 \sigma$ in Fig. $7 \mathrm{C}$. Omit map is contoured at $2.0 \sigma$ as shown in Fig. 7D. $\left(2 \mathrm{~F}_{\mathrm{o}}-\mathrm{F}_{\mathrm{c}}\right.$ map at $1 \sigma$ and omit map at $2.5 \sigma$ are shown in Fig. S9). The binding of TC does not cause major conformational change of VcDsbA. The complex has an r.m.s.d. value of $0.33 \AA$ over $143 \mathrm{C}^{\alpha}$ positions compared to apo VcDsbA (PDB ID 4DVC). ${ }^{[17]}$ The hydrophobic groove of $\mathrm{VcDsbA}$ is truncated and flatter than the groove of $\mathrm{EcDsbA}$ as a result of a three-residue deletion in loop L3 and a four-residue deletion in helix H7 (Fig. 1A-B). The VcDsbA-TC complex shows that TC binds only to the right-hand side of the VcDsbA groove where it makes 
hydrophobic contacts with A164, K165, S166 and L167 of loop L3 (Fig. 7A, B). This results in the polar end of TC binding directly under $\mathrm{H} 32$ of the active site. Its amide oxygen forms a hydrogen bond with the imidazole NH of $\mathrm{H} 32$ and the sulfonate forms a network of hydrogen bonds to the $\mathrm{OH}$ of Y170 and the backbone amide NH of G161. Close inspection of the crystal structure also showed that TC makes additional hydrophobic contacts with R107 and D121 of a symmetry-related molecule in the crystal lattice (Fig. S10). The binding site of TC in the costructure is further supported by the solution NMR data. As illustrated in Fig. 4, residues Q162, A164, K165, L167 and Y170 of VcDsbA, which are strongly perturbed upon addition of TC in the HSQC spectra, are all located within $5 \AA$ of TC in the crystal structure.

\section{Discussion}

Many human pathogens, including $V$. cholerae, Shigella flexneri, Neisseria meningitidis, Salmonella enterica, and uropathogenic E. coli (UPEC), rely on Dsb redox systems to deploy virulence proteins such as adhesion factors, secretion systems and toxins required for infection. ${ }^{[6,26]}$ As DsbA is not essential for bacterial viability, ${ }^{[27]}$ DsbA inhibitors are not expected to impair bacterial growth, which should reduce the pressure for selection of resistant strains and potentially preserve native microbiota. ${ }^{[28]}$ A recent study has shown that DsbA inhibitor resistance was not induced under conditions that rapidly induced resistance to antibiotics in Salmonella enterica serovar Typhimurium. ${ }^{[29]}$ Additionally, since DsbA is localized in the bacterial periplasm, access to the protein for inhibition by small molecules is expected to be less difficult than inhibition of cytoplasmic targets. These factors make DsbA a promising bacterial target for the development of antibacterial drugs. ${ }^{[6]}$

DsbA enzymes are widespread, although there are important differences among the DsbA systems that exist in different bacteria. ${ }^{[26 a]}$ Based on the surface charge and architecture surrounding the active site, DsbA proteins have been categorized into different subclasses, and those from E. coli, Salmonella enterica (SeDsbA), Klebsiella pneumoniae (KpDsbA) and V. cholerae have been classified into a common subclass, Ia. ${ }^{[30]}$ Given their high degree of structural similarity and the broadly conserved architecture of their hydrophobic grooves, it was postulated that DsbA enzymes in this subclass may be able to be targeted by subclass Iaspecific small molecule inhibitors. ${ }^{[30-31]}$ The hypothesis has been supported by studies showing that compounds designed to block EcDsbA could also inhibit KpDsbA and SeDsbA. ${ }^{[15 f, 31]}$ $\mathrm{KpDsbA}$ and SeDsbA share $>80 \%$ sequence identity with EcDsbA. In contrast, the sequences 
of EcDsbA and VcDsbA are less conserved and share only 39\% sequence identity (72/186 residues). Nonetheless, their structures are similar and can be superimposed with an r.m.s.d. of $1.3 \AA$ over $174 \mathrm{C}^{\alpha}$ atoms. ${ }^{[32]}$ Although they share similar surface features, the hydrophobic groove of $\mathrm{VcDsbA}$ is significantly truncated compared to that of EcDsbA, which presents a smaller surface area for interaction with substrates and small molecules (Fig. 1). In this work, we performed an NMR-based fragment screen against oxidized VcDsbA and identified benzimidazole 1 as a hit. Analogue screening then gave benzimidazole 2, which displayed a higher affinity for $\mathrm{VcDsbA}$ and was found to have $>8$-fold selectivity for $\mathrm{VcDsbA}$ over EcDsbA. This suggests the development of DsbA subclass Ia-specific inhibitors might not be readily extended to $\mathrm{VcDsbA}$. To rationalize the selectivity of this compound, we attempted to generate the structures of benzimidazole 2 in complex with both $\mathrm{VcDsbA}$ and EcDsbA. Due to the weak binding affinity of the compound to EcDsbA, we were unable to generate a model for the EcDsbA-benzimidazole 2 complex. The model of the VcDsbA complex shows that benzimidazole 2 binds to the hydrophobic groove of VcDsbA. Comparison of the NMR structure of benzimidazole 2 bound to VcDsbA with the apo crystal structure of EcDsbA (PDB $1 F V K)$ provides a potential explanation for the observed selectivity. An overlay of these two structures indicates that a similar mode of binding is not possible in EcDsbA as it would result in a steric clash between the benzimidazole ring and F174 and F36 side chains of EcDsbA.

TC is a component of bile salts secreted in the gastrointestinal tract and has been reported to bind to and inhibit both VcDsbA and EcDsbA. ${ }^{[18]}$ TC binds to VcDsbA with slightly greater affinity than EcDsbA. Therefore, TC offered an opportunity to elucidate a structural basis for the binding specificity observed for these two subclass Ia DsbA enzymes. We characterized the interactions of TC with VcDsbA and EcDsbA by NMR and X-ray crystallography and confirmed the slight selectivity for VcDsbA over EcDsbA in biophysical binding assays. The crystal structures revealed that TC adopts different binding poses in complex with EcDsbA and VcDsbA. Comparison of these two structures showed that the 7-residue deletion that causes truncation of the hydrophobic groove in VcDsbA significantly influences the binding pose of TC (Fig. 6,7). The side chains of F174, T168 and M171 in EcDsbA are oriented towards the active site, which narrows the right-hand side of the hydrophobic groove forming a small hydrophobic pocket around TC, pushing TC further towards the left-hand end of the groove where additional interactions are formed. In comparison, the side chains of VcDsbA residues S166 and K165, which are at similar spatial positions to F174 and T168 in EcDsbA, are oriented away from the active site, producing a more open right-hand side of the groove 
allowing the steroid core of TC to form hydrophobic contacts with the hydrophobic side chains of residues L167 and Y170. As a result, whilst the steroid core of TC interacts with residues in loop $\mathrm{L} 3$ of $\mathrm{VcDsbA}$, the more open conformation results in the molecule binding towards the right-hand side of the hydrophobic groove. In addition to hydrophobic interactions, the taurine portion of TC engages in a number of polar interactions. The VcDsbA-TC interface consists of three direct polar interactions to the backbone amide of Gly161 and the side chains of His 32 and Tyr170, whereas the EcDsbA-TC interface has two direct polar interactions to the backbone of Pro151 and the side chain of Gln160. In addition, there are a number of watermediated polar interactions in each complex (Fig. 8). These differences may contribute to the moderate binding selectivity of TC for VcDsbA over EcDsbA.

Comparison of the crystal structure of VcDsbA-TC complex and the NMR model of VcDsbAbenzimidazole 2 complex shows the benzimidazole overlays with the taurine end of the TC directly under H32, suggesting this site could be a hot spot for interaction with VcDsbA (Fig. 8A). The structural comparison of TC and benzimidazole 2 binding modes indicates expansion at the 2 or 3 -position of benzimidazole 2 with hydrophobic moieties may make additional hydrophobic contact with L3 similar to the steroid core of TC. In addition, the polar interactions that TC makes with $\mathrm{H} 32$ and P151 could potentially be exploited in the benzimidazole series through replacement of the trifluoromethyl and further expansion of the core (Fig. 8). Comparison of the crystal structure of TC bound to EcDsbA with previously reported costructures of EcDsbA in complex with diaryl ether $3^{[25]}$ shows the steroid core of TC makes similar hydrophobic interactions to diaryl ether 3 in the hydrophobic groove. TC reveals opportunities for expansion of these ligands to make similar polar interaction with Q160 and structural waters that may prove useful for designing diaryl ether analogues of higher affinity. Based on the current selectivity data, it is also anticipated that DsbA inhibitors that bind with similar affinity to EcDsbA and VcDsbA will be difficult to design.

The structures of TC in complex with two DsbA proteins provide structural data to facilitate our efforts to develop higher affinity small molecule inhibitors. However, the biological relevance of the ability of DsbA to bind TC is potentially interesting in its own right. When $E$. coli or $V$. cholera enter the gastrointestinal tract, they encounter a high concentration of bile salts. Small molecules with molecular weight $<700 \mathrm{Da}$, such as TC in this case, can access the periplasm via porins of the outer membrane, where they would encounter DsbA. ${ }^{[33]}$ Bile salts are known to be hijacked as host signals to activate $V$. cholerae virulence. ${ }^{[34]}$ It has been 
suggested that the binding of TC to VcDsbA induces virulence gene expression. ${ }^{[18,35]}$ In addition, an E.coli periplasmic protein UgpB has recently reported to act as a molecular chaperone by inhibiting protein aggregation induced by the bile salt cholate. ${ }^{[36]}$ UgpB contains a deep surface groove which potentially binds to bile salts. Based on our structures, it is possible that DsbA enzymes could also exhibit an anti-aggregation activity by directly binding to the bile salt TC. Therefore, DsbA may have multifaceted roles in mediating bacterial virulence and fitness in addition to its classic disulfide bond formation activity.

In summary, fragment hits identified through an NMR-based screen of VcDsbA, and analogue screening resulted in identification of benzimidazole $\mathbf{2}$, which was found to bind to the hydrophobic groove of $\mathrm{VcDsbA}$ with affinity $K_{\mathrm{D}}=446 \pm 10 \mu \mathrm{M}$. Although the binding affinity of this compound is weak, our NMR model of benzimidazole 2 in complex with VcDsbA provides a structural rationale for its observed selectivity and serves as a template for future inhibitor development. Benzimidazole 2 shows $>8$-fold selectivity for VcDsbA over EcDsbA despite the overall similarity of these two proteins. To better understand the structural basis of DsbA ligand binding selectivity, we also solved co-crystal structures of TC with both VcDsbA and EcDsbA. These DsbA:TC complex structures revealed markedly different TC binding modes, which appeared to be driven by truncation of the hydrophobic groove in VcDsbA. Comparison of these NMR and X-ray structures uncovered the molecular basis underlying selectivity of TC and benzimidazole 2 as well as suggesting potential strategies for the development of higher affinity binders.

\section{Materials and Methods}

Expression of unlabelled VcDsbA and EcDsbA. Unlabelled VcDsbA and EcDsbA were expressed by autoinduction as previously described. ${ }^{[15 b, 37]}$ Pre-expression cultures were prepared by inoculating $10 \mathrm{~mL}$ Luria-Bertani (LB) broth supplemented with $50 \mathrm{mg} / \mathrm{L}$ kanamycin from frozen glycerol stocks of E. coli BL21(DE3) carrying the plasmid coding for $\mathrm{VcDsbA}$ or EcDsbA and incubating $16 \mathrm{~h}$ at $37{ }^{\circ} \mathrm{C}$ with agitation at $220 \mathrm{rpm}$. ZYM-5052 media, ${ }^{[38]}$ using LB in place of ZYM, supplemented with $50 \mathrm{mg} / \mathrm{L}$ kanamycin in baffled conical flasks was inoculated with $1 \% \mathrm{v} / \mathrm{v}$ of pre-culture and incubated $24-30 \mathrm{~h}$ at $37{ }^{\circ} \mathrm{C}$ with agitation at $170 \mathrm{rpm}$, after which the cells were harvested by centrifugation at $3200 \mathrm{x} \mathrm{g} / 4{ }^{\circ} \mathrm{C}$ for $20 \mathrm{~min}$. The supernatant was discarded and the pellet was stored at $-20{ }^{\circ} \mathrm{C}$ for subsequent protein extraction and purification. 
Expression of uniformly ${ }^{15} \mathrm{~N}$-labeled DsbA. ${ }^{15} \mathrm{~N}$-labeled VcDsbA and EcDsbA were expressed by autoinduction. ${ }^{[38]} 20 \mathrm{x}^{15} \mathrm{NPS}, 50 \times 5052,1 \mathrm{M} \mathrm{MgSO}_{4}, 100 \mathrm{mg} / \mathrm{mL}$ thiamine and 1000x trace metals were prepared beforehand. Bacterial growth and expression media were prepared immediately prior to inoculation. Pre-expression cultures were prepared by inoculating $10 \mathrm{~mL} \mathrm{LB}$ broth supplemented with $50 \mathrm{mg} / \mathrm{L}$ kanamycin from frozen glycerol stocks of E. coli BL21(DE3) carrying the plasmid coding for VcDsbA or EcDsbA as appropriate and incubating $16 \mathrm{~h}$ at $37{ }^{\circ} \mathrm{C}$ with agitation at $220 \mathrm{rpm} .{ }^{15} \mathrm{~N}-5052$ media ${ }^{[38]}$ supplemented with $50 \mathrm{mg} / \mathrm{L}$ kanamycin in baffled conical flasks was inoculated with $1 \% \mathrm{v} / \mathrm{v}$ of pre-culture and incubated $24-30 \mathrm{~h}$ at $37^{\circ} \mathrm{C}$ with agitation at $170 \mathrm{rpm}$, after which the cells were harvested by centrifugation at $3200 \mathrm{xg} / 4^{\circ} \mathrm{C} / 20 \mathrm{~min}$. The supernatant was discarded and the pellet was stored at $-20{ }^{\circ} \mathrm{C}$ for subsequent protein extraction and purification.

Expression of uniformly labeled ${ }^{13} \mathbf{C},{ }^{15} \mathbf{N}$ DsbA. $400 \mathrm{~mL}$ of ${ }^{15} \mathrm{~N}-\mathrm{MG}$ growth media ${ }^{[38]}$ supplemented with $50 \mathrm{mg} / \mathrm{L}$ kanamycin in a 2-litre baffled conical flask was inoculated with 4 $\mathrm{mL}$ of appropriate pre-expression culture and incubated at $37{ }^{\circ} \mathrm{C}$ with agitation at $170 \mathrm{rpm}$. The optical density at $600 \mathrm{~nm}$ of the growth culture was monitored until $\mathrm{OD}_{600}$ approached 3.0. The growth culture was harvested by gentle centrifugation at $1800 \times \mathrm{g} / 20{ }^{\circ} \mathrm{C} / 15 \mathrm{~min}$, the supernatant was discarded and the cell pellet was gently resuspended in the ${ }^{13} \mathrm{C}^{15} \mathrm{~N}-\mathrm{MG} / \mathrm{Kan}^{+}$ expression media. This expression culture was transferred back to the baffled conical flask and incubated at $37{ }^{\circ} \mathrm{C}$ with agitation at $170 \mathrm{rpm}$. After $15 \mathrm{~min}$ for adaptation of the cells to the new media and to allow assimilation of ${ }^{13} \mathrm{C}$-labeled glucose, $1 \mathrm{mM}$ IPTG was added to induce expression and the culture was incubated a further $6 \mathrm{~h}$ at $37^{\circ} \mathrm{C}$ with agitation at $170 \mathrm{rpm}$, after which the cells were harvested by centrifugation at $3200 \mathrm{x} \mathrm{g} / 4^{\circ} \mathrm{C} / 20 \mathrm{~min}$. The supernatant was discarded and the pellet was stored at $-20^{\circ} \mathrm{C}$ for subsequent protein extraction and purification.

Protein extraction and purification. Each frozen bacterial pellet was thawed, resuspended in an equal volume of osmotic shock buffer (20 mM Tris ( $\mathrm{pH} 9.0), 10 \mathrm{mM}$ EDTA, 50\% w/v sucrose) and maintained at $4{ }^{\circ} \mathrm{C}$ with gentle stirring for $60 \mathrm{~min}$, after which the cell suspension was rapidly diluted to 10 times its volume with deionized $\mathrm{H}_{2} \mathrm{O}$ at $4{ }^{\circ} \mathrm{C}$. The suspension was maintained at $4{ }^{\circ} \mathrm{C}$ and stirred for a further $90 \mathrm{~min}$ before the cell pellet and lysate were separated by centrifugation at $50000 \times \mathrm{g} / 4{ }^{\circ} \mathrm{C} / 30 \mathrm{~min}$. The lysate was carefully decanted from the pellet and stored at $-20{ }^{\circ} \mathrm{C}$. The cell pellet was resuspended in 10 times its volume of lysis buffer (20 mM Tris (pH 8.0), $25 \mathrm{mM} \mathrm{NaCl}, 4 \mathrm{mg} / \mathrm{mL}$ colistin sulfate) and maintained at room temperature with gentle stirring for 18-24 h. This suspension was then centrifuged at $50000 \mathrm{x}$ 
$g / 4{ }^{\circ} \mathrm{C} / 30 \mathrm{~min}$ and the lysate was decanted and added to the thawed osmotic shock lysate. The pellet was discarded and the target protein was purified from the combined lysate. $\left(\mathrm{NH}_{4}\right)_{2} \mathrm{SO}_{4}$ was added to the combined lysate with gentle stirring to a concentration of $0.8 \mathrm{M}$ and the solution was syringe filtered (0.22 $\mu \mathrm{m}$, Millipore) for loading onto a HiLoad 1610 Phenyl Sepharose HP hydrophobic interaction chromatography column (GE Healthcare) using $20 \mathrm{mM}$ Tris ( $\mathrm{pH} 8.0), 50 \mathrm{mM} \mathrm{NaCl}, 1 \mathrm{M}\left(\mathrm{NH}_{4}\right)_{2} \mathrm{SO}_{4}$. The bound proteins were eluted with a $1-0 \mathrm{M}$ $\left(\mathrm{NH}_{4}\right)_{2} \mathrm{SO}_{4}$ gradient. Fractions were analyzed by SDS-PAGE and those containing target protein were pooled and concentrated to $10 \mathrm{~mL}$ using an Amicon centrifugal diafiltration unit (3000 MWCO, Millipore) and buffer exchanged to $50 \mathrm{mM}$ HEPES, $\mathrm{pH} 6.8,50 \mathrm{mM} \mathrm{NaCl}$ using a HiPrep 2610 desalting column (GE Healthcare). Following buffer exchange, the fractions containing target protein were pooled and loaded onto a MonoQ HR 10/10 anion-exchange column (GE Healthcare). In these buffer conditions, pure target protein was collected in the flowthrough fraction and impurities were eluted using a $50 \mathrm{mM}-1 \mathrm{M} \mathrm{NaCl}$ gradient. Protein purity was confirmed by SDS-PAGE.

Oxidation of DsbA. Following purification by anion-exchange chromatography, the target protein solution was concentrated by diafiltration (Amicon 3000 MWCO, Millipore) and treated with 10-molar excess of freshly prepared $15 \mathrm{mM}$ copper-phenanthroline solution. After $1 \mathrm{~h}$ reaction time at $0{ }^{\circ} \mathrm{C}$, the copper-phenanthroline was removed by buffer exchange using a HiPrep 2610 desalting column with Buffer A (50 mM HEPES, pH 6.8, $50 \mathrm{mM} \mathrm{NaCl}$ ).

Adjustment to final protein concentration. For NMR experiments, protein concentration was adjusted to $0.1 \mathrm{mM}$ by concentration using Amicon centrifugal diafiltration units (3000 MWCO, Millipore) or dilution using NMR buffer (50 mM HEPES, pH 6.8, $50 \mathrm{mM} \mathrm{NaCl}$ ). For CSP measurements using 1D ${ }^{1} \mathrm{H}$ and ${ }^{1} \mathrm{H}_{-}{ }^{15} \mathrm{~N}-\mathrm{HSQC}$ NMR, $\mathrm{D}_{2} \mathrm{O}$ was added to a final concentration of $10 \%(\mathrm{v} / \mathrm{v})$. For NOESY spectra of $\mathrm{VcDsbA}$ in the presence of benzimidazole 2, the protein sample was exchanged into NMR buffer (50 mM HEPES, pH 6.8, $50 \mathrm{mM} \mathrm{NaCl}$ ) containing $99 \% \mathrm{D}_{2} \mathrm{O}$. Final protein concentrations were quantified using a NanoVue UV spectrophotometer (GE Healthcare) based on an extinction coefficient for oxidized EcDsbA of $\varepsilon=28545 \mathrm{M}^{-1} \mathrm{~cm}^{-1}$, and for oxidized VcDsbA of $\varepsilon=10500 \mathrm{M}^{-1} \mathrm{~cm}^{-1}$.

Sequence specific resonance assignments of $\mathrm{VcDsbA}$ and EcDsbA. Backbone and side chain assignments of oxidized VcDsbA have been reported previously at $320 \mathrm{~K}$ (BMRB under accession number 7360) and $300 \mathrm{~K} \cdot{ }^{[37,39]}$ We further collected triple-resonance experiments (CBCAcoNH, HNCACB, HNCA, HNCO and HNCACO) at $298 \mathrm{~K}$ on a Bruker $600 \mathrm{MHz}$ 
NMR spectrometer equipped with CryoProbe to establish sequential assignments for VcDsbA through $\mathrm{C}^{\alpha}, \mathrm{C}^{\beta}$ and $\mathrm{C}$ shift correlation to backbone amides. $300 \mu \mathrm{L}$ of $0.3 \mathrm{mM}\left[\mathrm{U}-{ }^{13} \mathrm{C}^{15} \mathrm{~N}\right]-$ labelled VcDsbA (50 mM HEPES, pH 6.8, $50 \mathrm{mM} \mathrm{NaCl}, 2 \mathrm{mM}$ EDTA) in a $5 \mathrm{~mm}$ Shigemi tube was used for data acquisition. Data were processed using Topspin 3.2 (pl 7) and analyzed using CARA. ${ }^{[40]}$ Stereospecific assignment information for valine and leucine sidechain methyl groups was previously available ${ }^{[37]}$ and they were used for structure determination of VcDsbAbenzimidazole 2 complex using HADDOCK. We previously reported the backbone assignments of oxidized EcDsbA, ${ }^{[15 b]}$ and they were used for the chemical shift perturbation analysis in $2 \mathrm{D}{ }^{1} \mathrm{H}-{ }^{15} \mathrm{~N}$ HSQC spectra.

Fragment Screening of oxidized VcDsbA by STD NMR Spectroscopy. For ligand-based screening by STD NMR, unlabelled oxidized VcDsbA was prepared at $10 \mu \mathrm{M}$ in $10 \mathrm{mM}$ HEPES (pH 7.0), $150 \mathrm{mM} \mathrm{NaCl}$ buffer containing $10 \% \mathrm{D}_{2} \mathrm{O}$. A $550 \mu \mathrm{L}$ aliquot of this protein solution was added to each of one hundred screw-top Eppendorf tubes, each containing a cocktail comprising $1 \mu \mathrm{L}$ each of five Maybridge Library fragments in $\mathrm{D}_{6}$-DMSO. The final concentration of each fragment in the cocktail was $300 \mu \mathrm{M}$. STD spectra were acquired at 283 $\mathrm{K}$ on a Bruker Avance $800 \mathrm{MHz}$ spectrometer fitted with a triple-resonance $5 \mathrm{~mm}$ TXI Cryoprobe equipped with single-axis gradients and sample changer. Saturation was achieved by selective irradiation for a period of $3 \mathrm{~s}$ via a train of $50 \mathrm{~ms}$ Gaussian pulses centered at a frequency of -1 ppm. For the reference spectra a similar saturation pulse was applied $20000 \mathrm{~Hz}$ off-resonance. A $20 \mathrm{~ms}$ spin-lock period before acquisition allowed the protein signal to decay. The FID datasets were processed and the results were analyzed using Topspin by comparison of the STD spectra with reference 1D spectra of the individual compounds at $283 \mathrm{~K}$. The fragments that gave positive STDs in the cocktails were identified through alignment with their reference $1 \mathrm{D}$ spectra.

Hit validation and binding site identification using $2 \mathrm{D}{ }^{1} \mathrm{H}-{ }^{15} \mathrm{~N}$ HSQC NMR. Hits from the 1D ${ }^{1} \mathrm{H}$-STD NMR screen were validated, and the location of the fragment binding site was identified using $2 \mathrm{D}{ }^{1} \mathrm{H}-{ }^{15} \mathrm{~N}$ HSQC NMR. A reference ${ }^{1} \mathrm{H}^{-15} \mathrm{~N}$ HSQC of oxidized VcDsbA (100 $\mu \mathrm{M}\left[\mathrm{U}_{-}{ }^{15} \mathrm{~N}\right]$-labelled protein, $2 \% \mathrm{~d}_{6}$-DMSO, $50 \mathrm{mM}$ HEPES (pH 6.8), $50 \mathrm{mM} \mathrm{NaCl}$ ) was acquired and compared with a spectrum acquired under the same conditions for a sample containing the oxidized VcDsbA in the presence of a test fragment $(1 \mathrm{mM})$. In parallel, the effect of DMSO and $\mathrm{pH}$ on the chemical shifts of resonances in a sample of apo-VcDsbA were analysed to allow for identification of chemical shift perturbations caused by fragment binding. 
$3 \mathrm{~mm}$ thick-walled tubes of sample volume $\sim 160 \mu \mathrm{L}$ were used for NMR data collection. All data were acquired at $298 \mathrm{~K}$ on a Bruker $600 \mathrm{MHz}$ spectrometer equipped with auto-sampler and CryoProbe. Spectra were processed by Topspin, and analyzed either by $\mathrm{CARA}^{[40]}$ or SPARKY ${ }^{[41]}$. Weighted CSP were calculated using equation (1) ${ }^{[42]}$

$$
\operatorname{CSP}(\Delta \delta)=\sqrt{\Delta \delta_{H N}^{2}+\left(0.2 \times \Delta \delta_{N}\right)^{2}}
$$

where $\Delta \delta_{H N}$ and $\Delta \delta_{N}$ are the measured chemical shift difference of the backbone amide proton and nitrogen between apo- and holo-HSQC.

Affinity estimation from ligand-detected ${ }^{19} \mathrm{~F}$ NMR. The change in the resonance frequency of the single ${ }^{19} \mathrm{~F}$ signal in the $1 \mathrm{D}{ }^{19} \mathrm{~F}$ NMR spectrum of benzimidazole 2 was used to estimate the binding affinity using equation (2). ${ }^{[43]}$

$$
K_{D}=\frac{\gamma[L]_{1}-\gamma[L]_{2}}{1-\gamma}
$$

where $\gamma$ is described as $\Delta \delta \mathrm{F} 1 / \Delta \delta \mathrm{F} 2$ and $\Delta \delta \mathrm{F}_{1}$ and $\Delta \delta \mathrm{F}_{2}$ denote the changes in chemical shift of the fluorine signal of benzimidazole 2 in the absence of $\mathrm{VcDsbA}$ and in presence of $\operatorname{VcDsbA}(10.0 \mu \mathrm{M})$ at ligand concentrations of $[\mathrm{L}]_{1}$ and $[\mathrm{L}]_{2}$, respectively.

Analysis of VcDsbA-benzimidazole 2 complex using HADDOCK. The model structure of the VcDsbA-benzimidazole 2 complex was determined using the previously described approach. ${ }^{[21]}$ In brief, the PDB file (PDB ID: 4DVC) for VcDsbA was optimized using PDB_REDO ${ }^{[44]}$ and all water molecules, incidental ions and small molecules associated with crystallization were removed. The chain ID was removed and TER and END lines were added to each PDB file. Due to the dynamic nature of the hydrophobic pocket/groove in VcDsbA, an ensemble of protein conformers was generated from the crystal structure using a protocol described previously, ${ }^{[45]}$ and these were considered as the starting structures for HADDOCK calculations. ${ }^{[23,46]}$ Structure files for the fragments were prepared by $3 \mathrm{D}$ optimization in Maestro. ${ }^{[47]}$ A set of discrete ligand conformations was generated for benzimidazole 2 to account for ligand flexibility, using the ConfGen advanced panel in Maestro. ${ }^{[47]}$ The lowest energy conformations with similar potential energy values were retained and used for HADDOCK docking. Intermolecular distance restraints for VcDsbA-benzimidazole 2 complex were calibrated using the protocol described by Shah et al ${ }^{[46]}$ and are reported in Table S3. The restraints were tabulated in CNS format as input for HADDOCK. Electrostatics was defined as "on" throughout the structure calculations. On the basis of dynamics data for 
VcDsbA, ${ }^{[37]}$ the segment "162-166" was defined as fully flexible throughout HADDOCK docking. 1000 docked structures were calculated in the it0 iteration, 200 structures in the it1 iteration and then 200 structures were selected for a final flexible refinement in the explicit water iteration itw. At the end of each docking run, a family of 10 models of the complex with lowest HADDOCK score and restraint violation energy was examined manually in PyMOL.

X-ray data collection and structure determination. For the EcDsbA-TC complex, TC was dissolved in $100 \%$ methanol at a concentration of $100 \mathrm{mM} .10 \mu \mathrm{L}$ of this compound solution was mixed with $90 \mu \mathrm{L}$ of $15 \mathrm{mg} / \mathrm{mL}$ EcDsbA and incubated at $4{ }^{\circ} \mathrm{C}$ overnight. Crystals of the complex were grown at $20^{\circ} \mathrm{C}$ using the hanging drop vapor diffusion method from drops containing $1 \mu \mathrm{L}$ of the complex and $1 \mu \mathrm{L}$ of reservoir solution $(100-200 \mathrm{mM} \mathrm{KBr}, 28-33 \%$ PEG2000 MME). ${ }^{[15 b]}$ The crystals were cryo-protected by transferring to the reservoir solution and flashed-cooled in liquid nitrogen. For the VcDsbA-TC complex, TC powder (equivalent to $10 \mathrm{mM}$ final concentration) was directly added to $7 \mathrm{mg} / \mathrm{mL} \mathrm{VcDsbA}$ and incubated at $4{ }^{\circ} \mathrm{C}$ overnight. Crystals of the complex were grown at $20{ }^{\circ} \mathrm{C}$ using the hanging drop vapor diffusion method from drops containing $1.2 \mu \mathrm{L}$ of the complex and $0.8 \mu \mathrm{L}$ of reservoir solution $(0.1 \mathrm{M}$ Tris-HCl, $\mathrm{pH} 8.5,0.2 \mathrm{M} \mathrm{MgCl}_{2}, 26-36 \%$ PEG4000). The crystals were cryo-protected by transferring to a solution of 30\% PEG4000, $0.1 \mathrm{M}$ Tris-HCl, $\mathrm{pH} 8.5,15 \%$ glycerol and flashedcooled in liquid nitrogen.

Datasets were collected on the MX2 beamline at the Australian Synchrotron using Blue-Ice software. The MX2 beamline was equipped with an ADSC Quantum 315r detector. $1^{\circ}$ oscillation images were collected for a total of $180^{\circ}$. All datasets were indexed and integrated with $i M O S F L M^{[48]}$ and were scaled using AIMLESS. ${ }^{[49]}$ Phasing was performed by molecular replacement with Phaser MR using a previously solved structure of $E c$ DsbA (PDB code: 1FVK) as a search model. ${ }^{[50]}$ The structures were completed by iterative cycles of model building and refinement using Coot ${ }^{[51]}$ and Phenix. ${ }^{[52]}$ Data collection and refinement statistics are summarized in Table 1. Generation of molecular figures was carried out with PyMOL v1 7.2.1.

Isothermal Titration Calorimetry analysis. ITC was performed using a MicroCal $\mathrm{iTC}_{200}$ instrument (GE Healthcare). The sample cell was loaded with $250 \mu \mathrm{L}$ of oxidized DsbA at 100 $\mu \mathrm{M}$ in $25 \mathrm{mM}$ HEPES, $\mathrm{pH} 7.4,50 \mathrm{mM} \mathrm{NaCl}, 2 \%$ DMSO. The syringe was filled with $2 \mathrm{mM}$ sodium taurocholate (Sigma Aldrich) in the same buffer solution. Titrations were conducted at $25^{\circ} \mathrm{C}$ using 15 consecutive injections of $2.5 \mu \mathrm{L}$ with $180 \mathrm{~s}$ intervals and a stirring speed of 700 rpm. In every experiment, an initial $0.4 \mu \mathrm{L}$ of ligand was injected to avoid slow leakage of 
titrant and this data point was omitted from data analysis. The association constant $\left(K_{A}=1 / K_{\mathrm{D}}\right)$, enthalpy $(\Delta \mathrm{H})$, and entropy $(\Delta \mathrm{S})$ were calculated by fitting the data to a single-site binding model using the MicroCal Origin software. Every interaction was tested in triplicate and the values given represent mean and standard error of mean (SEM) from these triplicate analyses.

\section{ACCESSION NUMBERS}

Crystal structures of the EcDsbA-TC complex and the VcDsbA-TC complexes have been deposited into the Protein Data bank with code 7LUI and 7LSM, respectively.

\section{ACKNOWLEDGEMENTS}

This work was supported by the National Health and Medical Research Council (NHMRC) (Project Grants 1099151 \& 1144046). Dr Begoña Heras is supported by an ARC Future Fellowship (FT130100580). We would like to acknowledge the La Trobe UniversityComprehensive Proteomics Platform and the Monash Fragment Platform (MFP) for providing infrastructure and expertise. This research was undertaken using the MX1, MX2 beamlines at the Australian Synchrotron, part of ANSTO. Some of the NMR data were acquired at the NMR Facility of Bio21.

\section{References}

[1] M. Ali, A. R. Nelson, A. L. Lopez, D. A. Sack, PLoS Negl. Trop. Dis. 2015, 9, e0003832.

[2] (a) M. S. Islam, M. H. Zaman, M. S. Islam, N. Ahmed, J. D. Clemens, Vaccine 2019; (b) J. Reidl, K. E. Klose, FEMS Microbiol. Rev. 2002, 26, 125-139.

[3] S. J. Krebs, R. K. Taylor, J. Bacteriol. 2011, 193, 5260-5270.

[4] J. A. Peek, R. K. Taylor, Proc. Natl. Acad. Sci. U. S. A. 1992, 89, 6210-6214.

[5] D. X. Sun, M. J. Lafferty, J. A. Peek, R. K. Taylor, Gene 1997, 192, 79-85.

[6] B. Heras, M. J. Scanlon, J. L. Martin, Br. J. Clin. Pharmacol. 2015, 79, 208-215.

[7] M. Totsika, B. Heras, D. J. Wurpel, M. A. Schembri, J. Bacteriol. 2009, 191, 3901-3908.

[8] T. Miki, N. Okada, H. Danbara, J. Biol. Chem. 2004, 279, 34631-34642.

[9] aM. Watarai, T. Tobe, M. Yoshikawa, C. Sasakawa, Proc. Natl. Acad. Sci. U. S. A. 1995, 92, 4927-4931; bD. Missiakas, C. Georgopoulos, S. Raina, Proc. Natl. Acad. Sci. U. S. A. 1993, 90, 70847088.

[10] J. L. Martin, J. C. A. Bardwell, J. Kuriyan, Nature 1993, 365, 464-468. 
[11] J. J. Paxman, N. A. Borg, J. Horne, P. E. Thompson, Y. Chin, P. Sharma, J. S. Simpson, J. Wielens, S. Piek, C. M. Kahler, H. Sakellaris, M. Pearce, S. P. Bottomley, J. Rossjohn, M. J. Scanlon, J. Biol. Chem. 2009, 284, 17835-17845.

[12] S. Kishigami, E. Kanaya, M. Kikuchi, K. Ito, J. Biol. Chem. 1995, 270, 17072-17074.

[13] U. Grauschopf, A. Fritz, R. Glockshuber, EMBO J. 2003, 22, 3503-3513.

[14] M. R. Arkin, Y. Tang, J. A. Wells, Chem. Biol. 2014, 21, 1102-1114.

[15] (a) V. Fruh, Y. Zhou, D. Chen, C. Loch, E. Ab, Y. N. Grinkova, H. Verheij, S. G. Sligar, J. H. Bushweller, G. Siegal, Chem. Biol. 2010, 17, 881-891; (b) L. A. Adams, P. Sharma, B. Mohanty, O. V. Ilyichova, M. D. Mulcair, M. L. Williams, E. C. Gleeson, M. Totsika, B. C. Doak, S. Caria, K. Rimmer, J. Horne, S. R. Shouldice, M. Vazirani, S. J. Headey, B. R. Plumb, J. L. Martin, B. Heras, J. S. Simpson, M. J. Scanlon, Angew. Chem. Int. 2015, 54, 2179-2184; (c) C. Landeta, J. L. Blazyk, F. Hatahet, B. M. Meehan, M. Eser, A. Myrick, L. Bronstain, S. Minami, H. Arnold, N. Ke, E. J. Rubin, B. C. Furie, B. Furie, J. Beckwith, R. Dutton, D. Boyd, Nat. Chem. Biol. 2015, 11, 292-298; (d) M. A. Halili, P. Bachu, F. Lindahl, C. Bechara, B. Mohanty, R. C. Reid, M. J. Scanlon, C. V. Robinson, D. P. Fairlie, J. L. Martin, ACS Chem. Biol. 2015, 10, 957-964; (e) L. Duncan, Wang, G., Ilyichova, O, Scanlon, MJ., Heras, B., Abbott, B., Molecules 2019, 24; (f) M. Totsika, D. Vagenas, J. J. Paxman, G. Wang, R. Dhouib, P. Sharma, J. L. Martin, M. J. Scanlon, B. Heras, Antioxid. Redox Signal. 2017; (g) S. Nebl, W. S. Alwan, M. L. Williams, G. Sharma, A. Taylor, B. C. Doak, K. L. Wilde, R. M. McMahon, M. A. Halili, J. L. Martin, B. Capuano, R. B. Fenwick, B. Mohanty, M. J. Scanlon, J. Biomol. NMR 2020.

[16] W. Duprez, L. Premkumar, M. A. Halili, F. Lindahl, R. C. Reid, D. P. Fairlie, J. L. Martin, J. Med. Chem. 2015, 58, 577-587.

[17] P. M. Walden, B. Heras, K. E. Chen, M. A. Halili, K. Rimmer, P. Sharma, M. J. Scanlon, J. L. Martin, Acta. Crystallogr. D Biol. Crystallogr. 2012, 68, 1290-1302.

[18] Y. Xue, F. Tu, M. Shi, C. Q. Wu, G. Ren, X. Wang, W. Fang, H. Song, M. Yang, Mol. Microbiol. 2016, 102, 909-924.

[19] B. Mohanty, K. Rimmer, R. M. McMahon, S. J. Headey, M. Vazirani, S. R. Shouldice, M. Coincon, S. Tay, C. J. Morton, J. S. Simpson, J. L. Martin, M. J. Scanlon, PLoS One 2017, 12, e0173436.

[20] J. B. Jordan, D. A. Whittington, M. D. Bartberger, E. A. Sickmier, K. Chen, Y. Cheng, T. Judd, J. Med. Chem. 2016, 59, 3732-3749.

[21] B. Mohanty, M. L. Williams, B. C. Doak, M. Vazirani, O. Ilyichova, G. Wang, W. Bermel, J. S. Simpson, D. K. Chalmers, G. F. King, M. Mobli, M. J. Scanlon, J. Biomol. NMR 2016, 66, 195-208. [22] L. S. I. M. Robertson, B. D. Sykes, in Proceedings of the NATO Advanced Study Institute on Biophysics and the challenges of Emerging Threats, Springer, 2009, pp. 101-119.

[23] (a) C. Dominguez, R. Boelens, A. M. J. J. Bonvin, J. Am. Chem. Soc. 2003, 125, 1731-1737; (b) A. D. van Dijk, R. Boelens, A. M. Bonvin, FEBS J. 2005, 272, 293-312.

[24] C. M. Cremers, D. Knoefler, V. Vitvitsky, R. Banerjee, U. Jakob, P. Natl. Acad. Sci. U. S. A. 2014, 111, E1610-E1619. 
[25] M. R. Bentley, O. V. Ilyichova, G. Wang, M. L. Williams, G. Sharma, W. S. Alwan, R. L. Whitehouse, B. Mohanty, P. J. Scammells, B. Heras, J. L. Martin, M. Totsika, B. Capuano, B. C. Doak, M. J. Scanlon, J. Med. Chem. 2020, 63, 6863-6875.

[26] (a) B. Heras, S. R. Shouldice, M. Totsika, M. J. Scanlon, M. A. Schembri, J. L. Martin, Nat. Rev. Microbiol. 2009, 7, 215-225; (b) C. Landeta, D. Boyd, J. Beckwith, Nat. Microbiol. 2018, 3, 270280; (c) R. P. Smith, J. J. Paxman, M. J. Scanlon, B. Heras, Molecules 2016, 21 ; (d) C. Santos-Martin, G. Wang, P. Subedi, L. Hor, M. Totsika, J.J. Paxman, B. Heras, Comput. Struct. Biotechnol 2021, 19, 4725-4737.

[27] J. C. A. Bardwell, K. McGovern, J. Beckwith, Cell 1991, 67, 581-589.

[28] M. Totsika, Drug Deliv. Lett. 2016, 6, 30-37.

[29] R. Dhouib, D. Vagenas, Y. Hong, A. D. Verderosa, J. L. Martin, B. Heras, M. Totsika, FASEB Bioadv. 2021, 3, 231-242.

[30] R. M. McMahon, L. Premkumar, J. L. Martin, Biochim. Biophys. Acta. 2014, 1844, 1391-1401.

[31] F. Kurth, K. Rimmer, L. Premkumar, B. Mohanty, W. Duprez, M. A. Halili, S. R. Shouldice, B. Heras, D. P. Fairlie, M. J. Scanlon, J. L. Martin, PLoS One 2013, 8, e80210.

[32] S. H. Hu, J. A. Peek, E. Rattigan, R. K. Taylor, J. L. Martin, J. Mol. Biol. 1997, 268, 137-146.

[33] M. Masi, M. Refregiers, K. M. Pos, J. M. Pages, Nat. Microbiol. 2017, 2, 17001.

[34] A. J. Hay, J. Zhu, Infect. Immun. 2015, 83, 317-323.

[35] M. Yang, Z. Liu, C. Hughes, A. M. Stern, H. Wang, Z. Zhong, B. Kan, W. Fenical, J. Zhu, Proc. Natl. Acad. Sci. U. S. A. 2013, 110, 2348-2353.

[36] C. Lee, P. Betschinger, K. Wu, D. S. Zyla, R. Glockshuber, J. C. Bardwell, EMBO J. 2020, e104231.

[37] J. Horne, E. J. d'Auvergne, M. Coles, T. Velkov, Y. Chin, W. N. Charman, R. Prankerd, P. R. Gooley, M. J. Scanlon, J. Mol. Biol. 2007, 371, 703-716.

[38] F. W. Studier, Protein expression and purification 2005, 41, 207-234.

[39] J. Horne, M. J. Scanlon, Biomol. NMR Assign. 2007, 1, 75-76.

[40] R. L. J. Keller, The computer aided resonance assignment tutorial 2004.

[41] W. Lee, M. Tonelli, J. L. Markley, Bioinformatics 2015, 31, 1325-1327.

[42] J. J. Ziarek, F. C. Peterson, B. L. Lytle, B. F. Volkman, Methods Enzymol 2011, 493, 241-275.

[43] J. B. Jordan, L. Poppe, X. Xia, A. C. Cheng, Y. Sun, K. Michelsen, H. Eastwood, P. D. Schnier, T. Nixey, W. Zhong, J. Med. Chem. 2012, 55, 678-687.

[44] R. P. Joosten, F. Long, G. N. Murshudov, A. Perrakis, IUCrJ 2014, 1, 213-220.

[45] B. Mohanty, P. Serrano, B. Pedrini, K. Jaudzems, M. Geralt, R. Horst, T. Herrmann, M. A. Elsliger, I. A. Wilson, K. Wuthrich, Acta. Crystallogr. Sect. F Struct. Biol. Cryst. Commun. 2010, 66, 1381-1392.

[46] D. M. Shah, E. Ab, T. Diercks, M. A. S. Hass, N. A. J. van Nuland, G. Siegal, J. Med. Chem. 2012, 55, 10786-10790. 
[47] I. Maestro Schrodinger, 9.7 ed., New York, NY, 2014.

[48] T. G. Battye, L. Kontogiannis, O. Johnson, H. R. Powell, A. G. Leslie, Acta. Crystallogr. D Biol. Crystallogr. 2011, 67, 271-281.

[49] P. R. Evans, G. N. Murshudov, Acta. Crystallogr. D Biol. Crystallogr. 2013, 69, 1204-1214.

[50] L. W. Guddat, J. C. A. Bardwell, R. Glockshuber, M. HuberWunderlich, T. Zander, J. L. Martin, Protein Sci. 1997, 6, 1893-1900.

[51] P. Emsley, K. Cowtan, Acta. Crystallogr. D Biol. Crystallogr. 2004, 60, 2126-2132.

[52] P. D. Adams, R. W. Grosse-Kunstleve, L. W. Hung, T. R. Ioerger, A. J. McCoy, N. W. Moriarty, R. J. Read, J. C. Sacchettini, N. K. Sauter, T. C. Terwilliger, Acta. Crystallogr. D Biol. Crystallogr. 2002, 58, 1948-1954. 

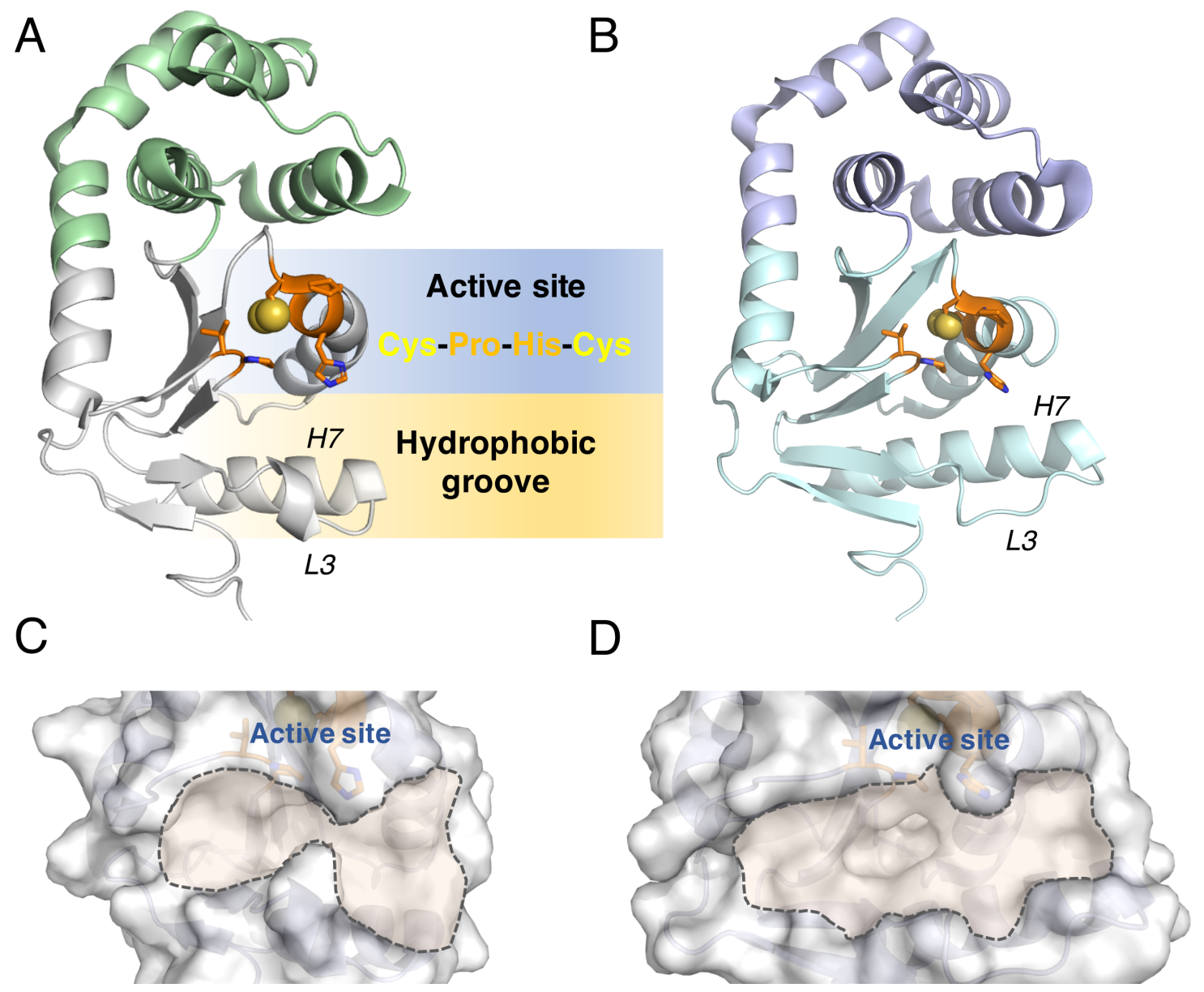

Figure 1. Crystal structures of VcDsbA and EcDsbA. A. Crystal structure of oxidized VcDsbA (PDB code: 4DVC). The thioredoxin domain is coloured in white, $\alpha$-helical domain is coloured in green. $\alpha$-helix H7 and loop L3, which form a part of the hydrophobic groove are labelled in the structure. Active site residues V148, cis-P149, C30, P31, H32 and C33 are shown as orange sticks. Sulfur atoms of redox active cysteines are shown as spheres. B. Crystal structure of oxidized EcDsbA (PDB code: $1 \mathrm{FVK}$ ). The thioredoxin domain is coloured in cyan, $\alpha$-helical domain is coloured in purple. $\alpha$-helix H7 and loop L3, which form a part of the hydrophobic groove, are labelled in the structure. Active site residues V150, cis-P151, C30, P31, H32 and C33 are shown as orange sticks. Sulfur atoms of redox active cysteines are shown as spheres. C-D. The hydrophobic grooves of VcDsbA and EcDsbA are highlighted by dashed lines on the surface models. The active sites are labelled in the structures. 
A<smiles>OCCCc1nc2ccc(C(F)(F)F)cc2[nH]1</smiles>
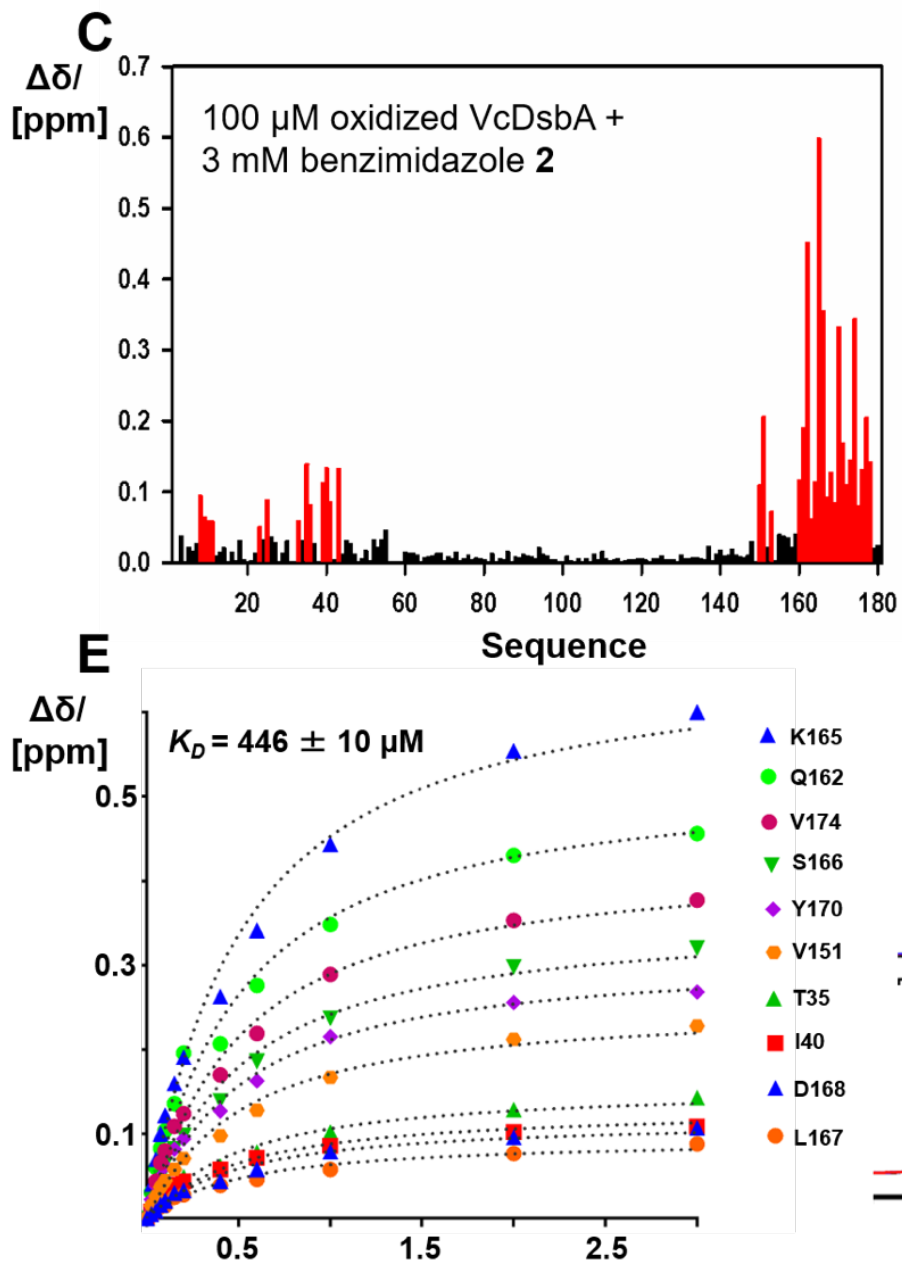

[Benzimidazole 2] (mM)<smiles>OCCCc1nc2c(Cl)cc(C(F)(F)F)cc2[nH]1</smiles>

D

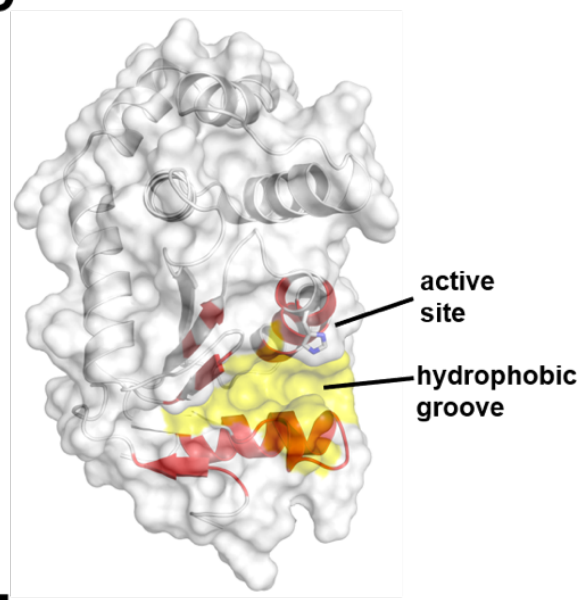

$\mathbf{F}$

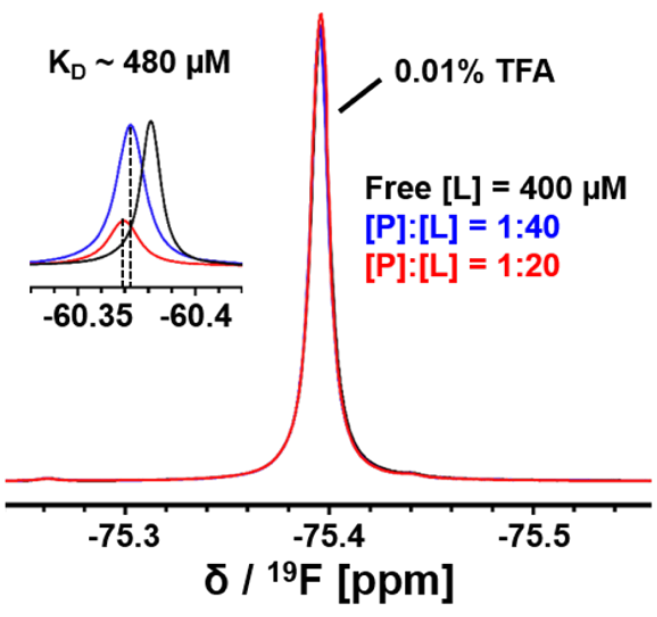

Figure 2. Characterization of benzimidazole 2 binding to VcDsbA. A. Chemical structure of the initial hit benzimidazole 1. B. Chemical structure of the best benzimidazole $\mathbf{2}$. C. Histogram showing chemical shift perturbations (CSP) observed in the ${ }^{1} \mathrm{H}-{ }^{15} \mathrm{~N}$ HSQC spectrum of VcDsbA $(100 \mu \mathrm{M})$ upon addition of $3 \mathrm{mM}$ benzimidazole 2 . Residues with CSP greater than 0.05 ppm are shown in red D. Residues with large CSP are coloured red and mapped onto the crystal structure of VcDsbA. The protein surface formed by hydrophobic groove residues is shown in yellow. E. CSP were measured with increasing concentrations of benzimidazole 2 to estimate the binding affinity $\left(K_{\mathrm{D}}\right)$. F. 1D ${ }^{19}$ F-NMR spectra of benzimidazole 2 at different concentration ratios of unlabelled oxidized VcDsbA and benzimidazole 2 . VcDsbA 
concentration was held constant at $10 \mu \mathrm{M}$ and the benzimidazole 2 concentration was varied as indicated. Spectra were referenced and normalized to the resonance of an internal standard of trifluoroacetic acid $(0.01 \% v / v)$. 


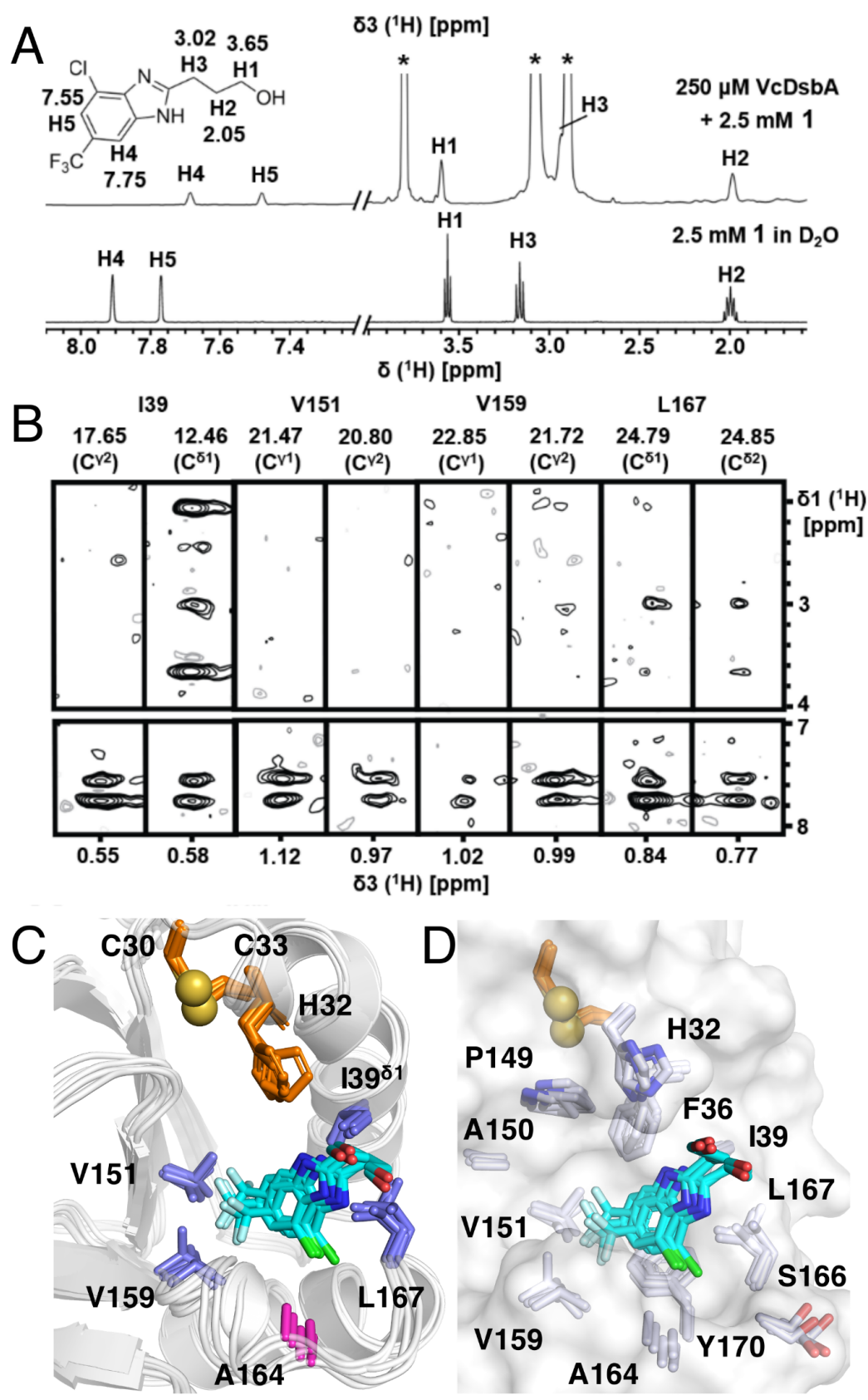

Figure 3. HADDOCK docking model of oxidized VcDsbA in complex with benzimidazole

2. A. Structure of benzimidazole 2 with ${ }^{1} \mathrm{H}$ NMR assignments in the presence (top) and absence (bottom) of oxidized VcDsbA. * Indicates buffer signals. B. $2 \mathrm{D}\left[{ }^{1} \mathrm{H},{ }^{1} \mathrm{H}\right]-\mathrm{NOESY}$ strip plot of 3D $\omega 1-{ }^{13} \mathrm{C},{ }^{15} \mathrm{~N}$-filtered, $\omega 3-{ }^{13} \mathrm{C}_{\mathrm{ali}}$ (methyl) edited $\left[{ }^{1} \mathrm{H},{ }^{1} \mathrm{H}\right]-\mathrm{NOESY}$ spectrum of oxidized $\mathrm{VcDsbA}$ in the presence of benzimidazole 2. Strips are shown for residues where intermolecular NOEs with benzimidazole 2 were observed. C. Five lowest-energy HADDOCK models of the VcDsbA-benzimidazole 2 complex. The protein is shown as a cartoon and the ligand in stick representation. Residues for which intermolecular NOEs with benzimidazole 2 were observed are shown in blue sticks and labelled. No intermolecular NOEs were observed from benzimidazole 2 to $\mathrm{A} 164-\mathrm{CH}_{3}$, this residue is located at the lower edge of hydrophobic 
groove of VcDsbA. The side chain of A164 is shown in magenta. D. Surface representation of $\mathrm{VcDsbA}$ in the same orientation as (C) with residues within $5 \AA$ of benzimidazole 2 shown as sticks. In both panels $\mathrm{C}$ and $\mathrm{D}$ active site side chain residues are shown as orange sticks and the sulfur atoms of $\mathrm{C} 30$ and $\mathrm{C} 33$ as yellow spheres. 


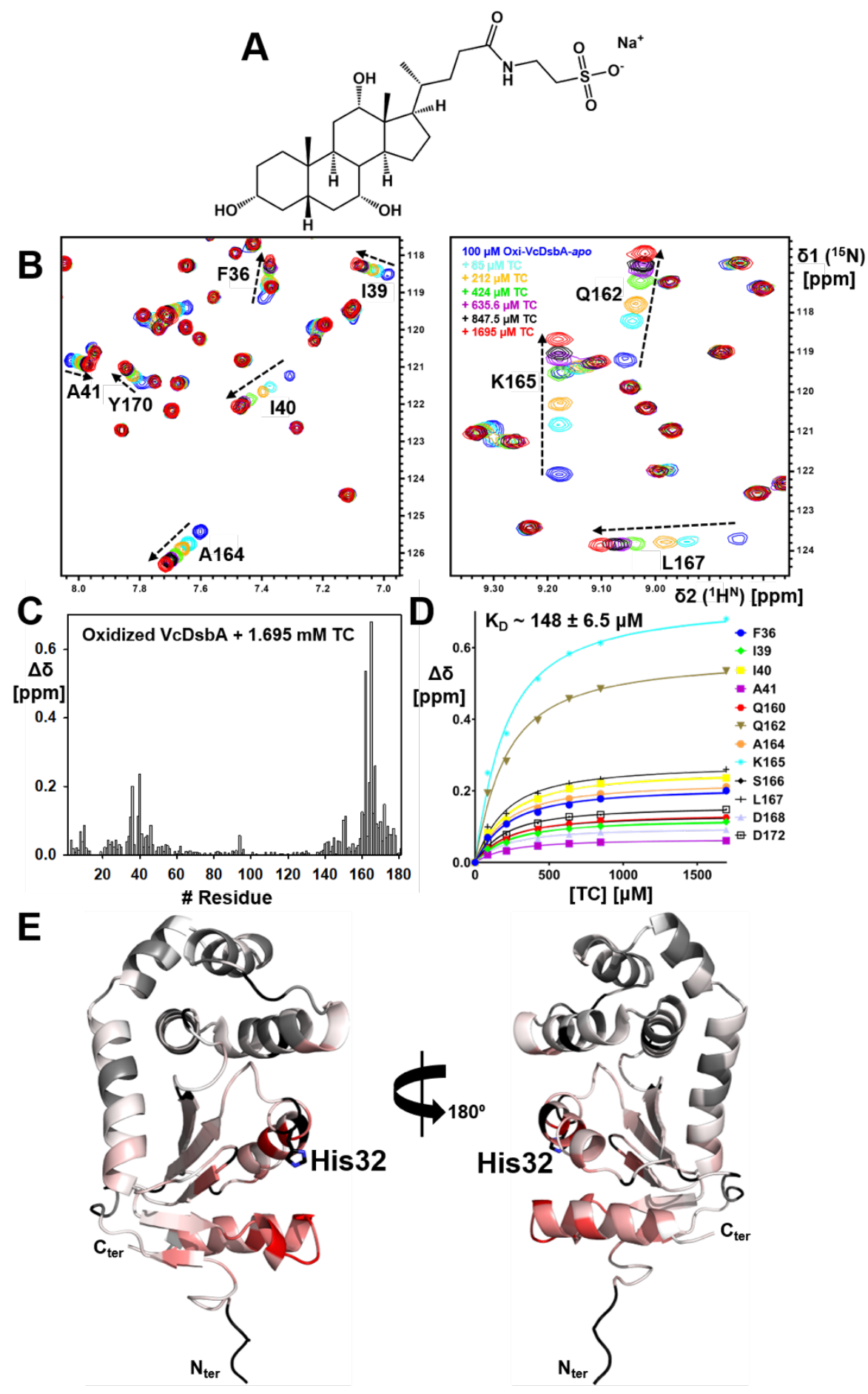

Figure 4. Chemical shift perturbations (CSP) observed in the ${ }^{1} \mathrm{H}-{ }^{15} \mathrm{~N}$ HSQC spectrum of oxidized VcDsbA upon addition of sodium taurocholate (TC). A. Chemical structure of sodium taurocholate. B. Chemical shift perturbations (CSP) observed in the ${ }^{1} \mathrm{H}^{-1}{ }^{15} \mathrm{NSQC}$ spectrum of VcDsbA with increasing concentrations of TC. TC concentrations are shown in the inset in the same colour as the corresponding spectrum. C. CSP histogram plot of oxidized VcDsbA $(100 \mu \mathrm{M})$ upon addition of $1.7 \mathrm{mM}$ TC. D. The concentration-dependent CSP were 
fitted to a one-site binding model to estimate the binding affinity $\left(K_{\mathrm{D}}\right)$. E. CSP are mapped onto the crystal structure of $\mathrm{VcDsbA}$ (PDB ID: 4DVC) and are shown in colour gradient red (CSP $=0.2 \mathrm{ppm})$ to white $(\mathrm{CSP}=0 \mathrm{ppm})$. Active site $\mathrm{H} 32$ residue is shown as sticks. Unassigned residues are shown in black. 


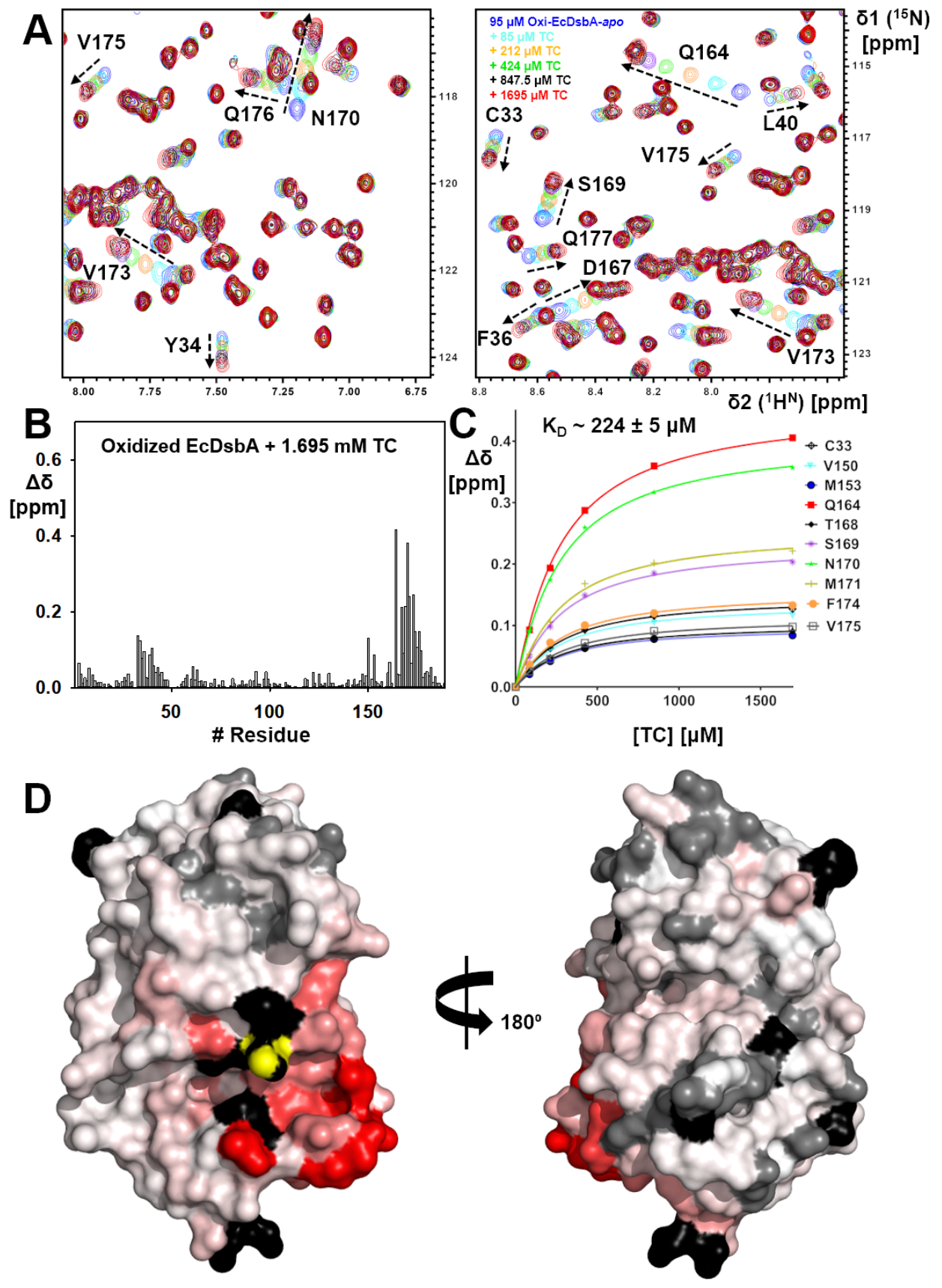

Figure 5. Chemical shift perturbations (CSP) observed in the ${ }^{1} \mathrm{H}-{ }^{15} \mathrm{~N}$ HSQC spectrum of oxidized EcDsbA upon addition of sodium taurocholate (TC). A. Chemical shift perturbations (CSP) observed in the ${ }^{1} \mathrm{H}-{ }^{15} \mathrm{~N}$ HSQC spectrum of EcDsbA with increasing concentrations of TC. TC concentrations are shown in the inset in the same colour as the corresponding spectrum. B. CSP histogram plot of oxidized EcDsbA $(100 \mu \mathrm{M})$ upon addition of $1.695 \mathrm{mM}$ TC. C. The concentration-dependent CSP were fitted to a one-site binding model to estimate the binding affinity $\left(K_{\mathrm{D}}\right)$. D. CSP were mapped onto the crystal structure of EcDsbA 
(PDB ID: 1FVK) and are shown in colour gradient red $(\mathrm{CSP}=0.2 \mathrm{ppm})$ to white $(\mathrm{CSP}=0$ ppm). Active site $\mathrm{H} 32$ residue is shown in yellow. Unassigned residues are shown in black. 

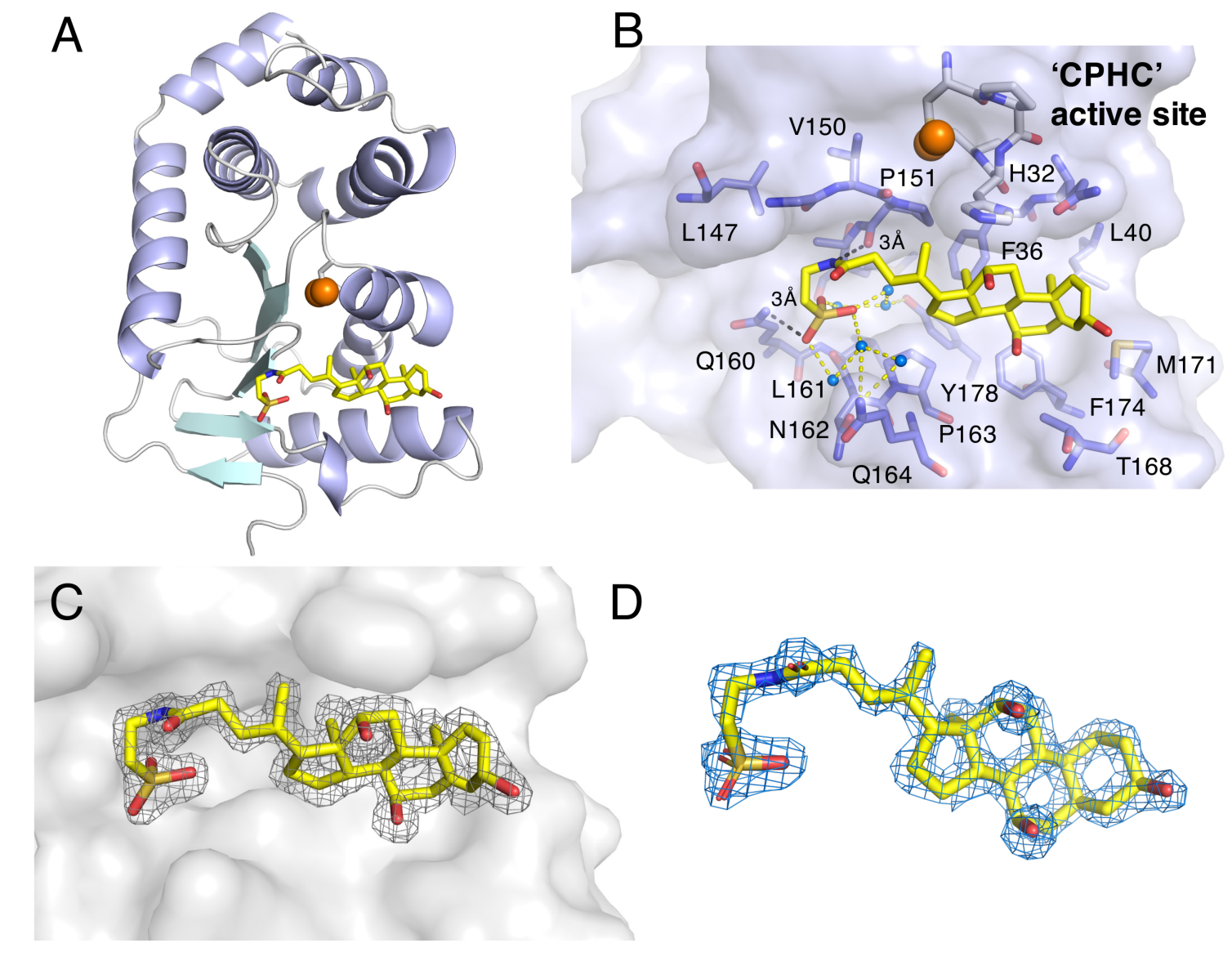

Figure 6. Crystal structure of oxidized EcDsbA in complex with sodium taurocholate (TC). A. Cartoon model of the EcDsbA-taurocholate complex. Sulfur atoms of redox active Cys30 and Cys33 are shown as orange spheres. $\alpha$-helices are shown in light purple and $\beta$ strands are shown in cyan. B. A cluster of residues within $5 \AA$ of taurocholate are shown as sticks and labelled. Sulfur atoms of redox active Cys30 and Cys33 are shown as orange spheres. CPHC active site is indicated in the structure. The distance of polar interactions is labelled and shown as black dashed lines. Water-mediated hydrogen bond network is shown as yellow dashed lines. C. $2 F_{\mathrm{o}}-\mathrm{F}_{\mathrm{c}}$ electron density map contoured at $1 \sigma$ is shown as grey mesh. EcDsbA is shown as grey surface. $\mathbf{D}$. Simulated annealing omit $\sigma_{\mathrm{A}}$-weighted $\mathrm{mF}_{\mathrm{o}}-\mathrm{DF}_{\mathrm{c}}$ electron density map contoured at $3.0 \sigma$ is shown as blue mesh. 

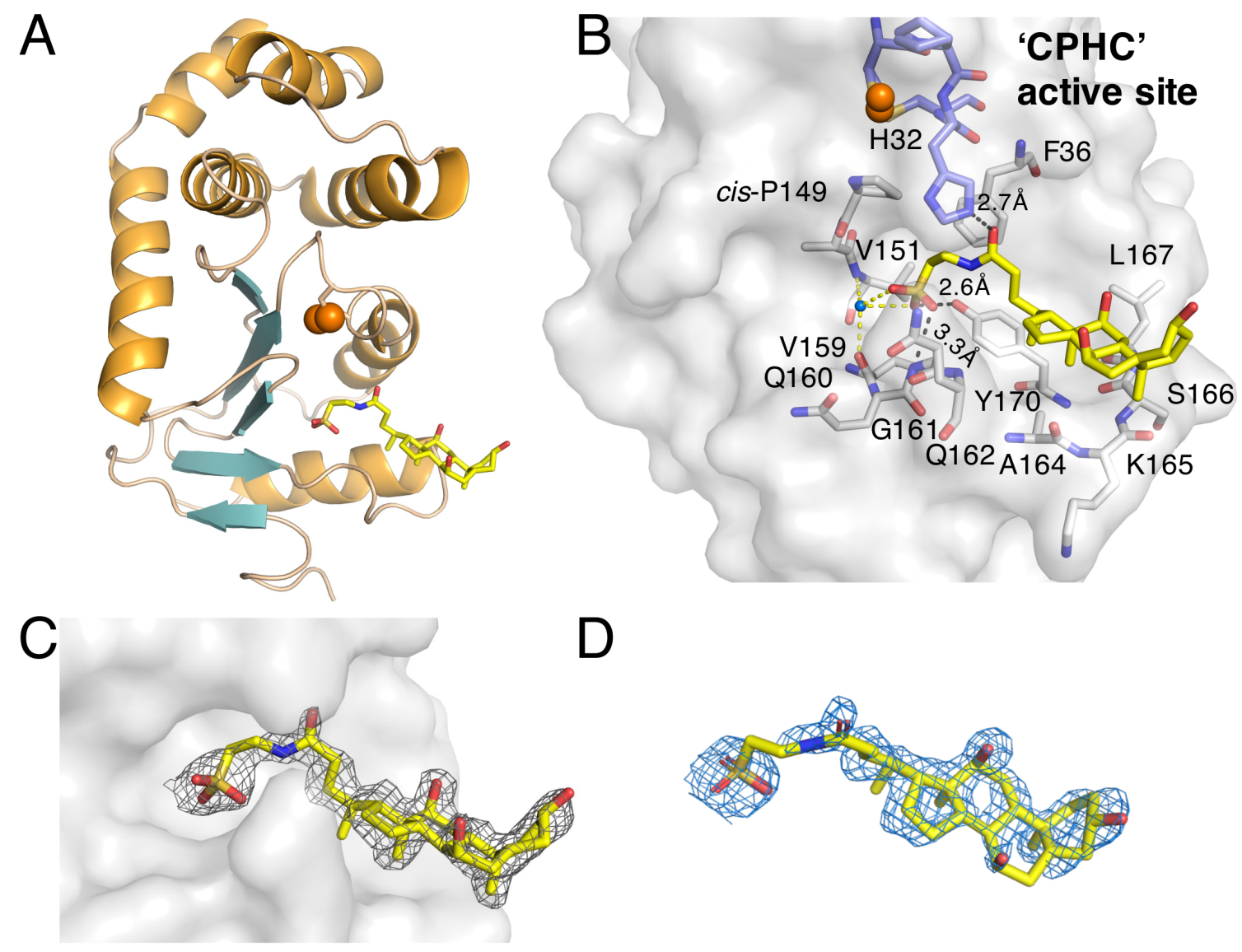

$\mathrm{D}$

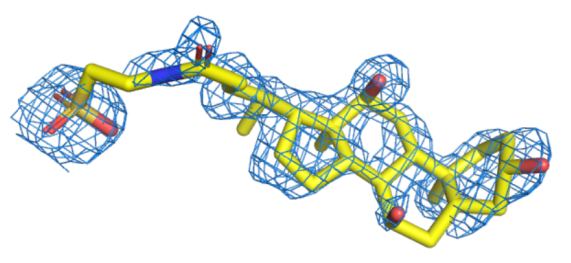

Figure 7. Crystal structure of oxidized VcDsbA in complex with sodium taurocholate (TC). A. Cartoon model of the VcDsbA-taurocholate complex. Sulfur atoms of redox active Cys30 and Cys 33 are shown as orange spheres. $\alpha$-helices are shown in orange and $\beta$-sheets are shown in cyan. B. A cluster of residues within $5 \AA$ of taurocholate is shown as sticks and labelled. The distance of polar interactions is shown as black dashed lines. Water-mediated polar interactions are indicated by yellow dashed lines. C. $2 F_{0}-F_{c}$ electron density map contoured at $0.7 \sigma$ is shown as grey mesh. VcDsbA is shown as grey surface. D. Simulated annealing omit $\sigma_{\mathrm{A}}$-weighted $\mathrm{mF}_{\mathrm{o}}-\mathrm{DF}_{\mathrm{c}}$ electron density map contoured at $2.0 \sigma$ is shown as blue mesh. 

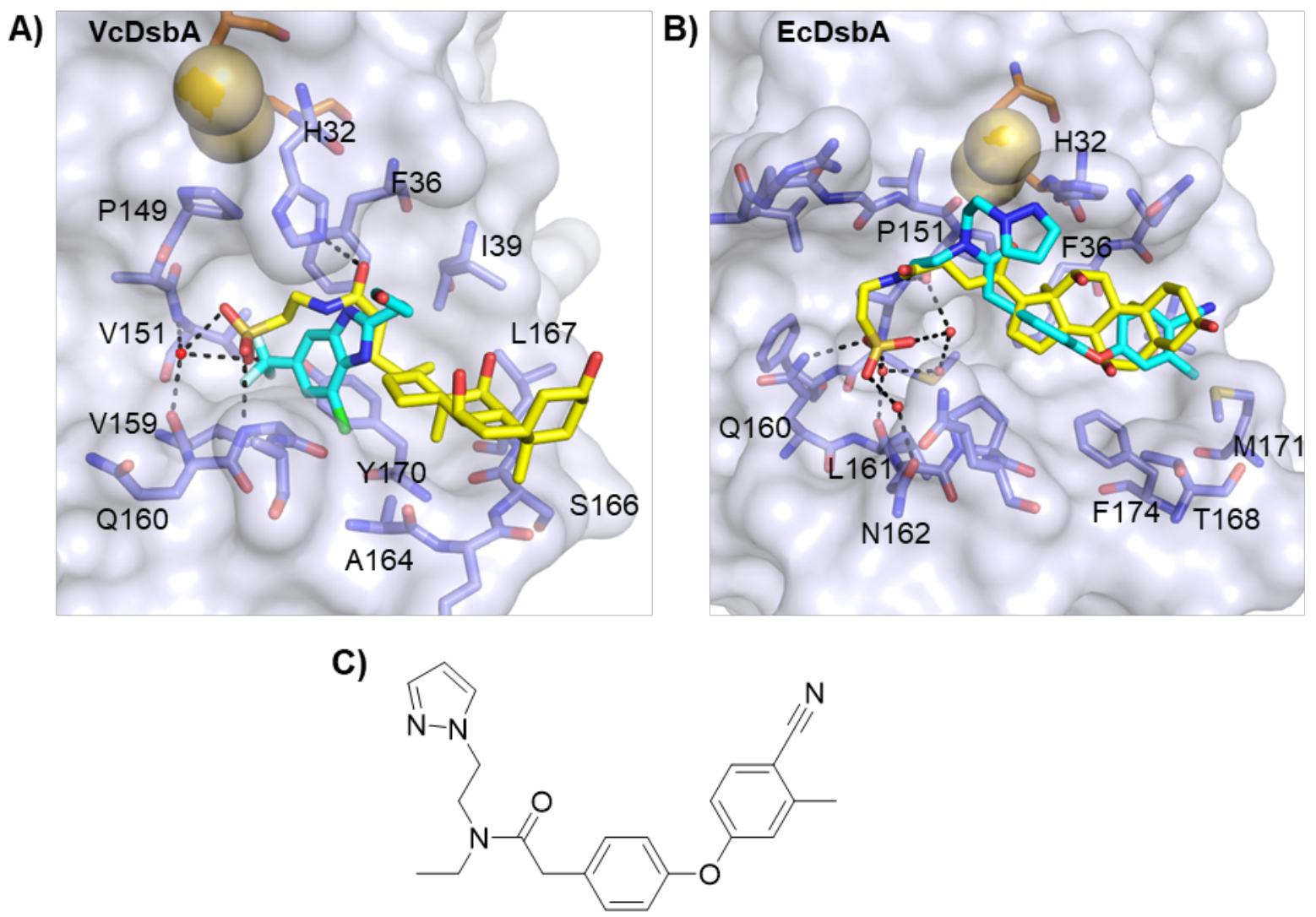

3

Figure 8. Comparison of binding poses of TC and DsbA inhibitors. A. Structure overlay of benzimidazole 2 (cyan sticks) with the VcDsbA (white surface, blue sticks)-TC (yellow stick) complex. B. Structural overlay of the complexes of diaryl ether 3 (cyan sticks) with the EcDsbA (white surface, blue sticks)-TC (yellow) complex (PDB ID:6PDH). In both cases the active site Cys30 and Cys33 residues are shown as orange sticks, sulfur atoms shown as yellow spheres. Residues within $6 \AA$ of TC are shown as blue sticks and interacting waters red spheres with key residues labelled. Polar contacts are shown as dashed black lines. C. Chemical structure of diaryl ether 3. 